PALEO

Revue d'archéologie préhistorique

$18 \mid 2006$

Varia

\title{
Les couches supérieures de la Micoque (Dordogne)
}

Upper levels of La Micoque (Dordogne)

\section{Gaëlle Rosendahl}

\section{OpenEdition}

\section{Journals}

Édition électronique

URL : http://journals.openedition.org/paleo/209

DOI : $10.4000 /$ paleo.209

ISSN : 2101-0420

Éditeur

SAMRA

\section{Édition imprimée}

Date de publication : 1 décembre 2006

Pagination : 161-192

ISSN : 1145-3370

\section{Référence électronique}

Gaëlle Rosendahl, « Les couches supérieures de la Micoque (Dordogne) », PALEO [En ligne], 18 | 2006, mis en ligne le 23 avril 2009, consulté le 07 juillet 2020. URL : http://journals.openedition.org/paleo/ 209 ; DOI : https://doi.org/10.4000/paleo.209

\section{(c) (i) (9)}

PALEO est mis à disposition selon les termes de la licence Creative Commons Attribution - Pas d'Utilisation Commerciale - Pas de Modification 4.0 International. 


\title{
LES COUCHES SUPÉRIEURES DE LA MICOQUE (Dordogne)
}

\author{
Gaëlle ROSENDAHL ${ }^{(1)}$
}

\begin{abstract}
Résumé : La couche 6 (N) du site de La Micoque, découvert en 1895, est la couche éponyme du Micoquien dont la définition varie fortement selon les auteurs. Cette couche 6 n'est cependant plus accessible sur le terrain et l'ancienneté des fouilles ainsi que la dispersion des pièces dans le monde entier rendent difficile une définition de son contenu. En outre, le système stratigraphique d'un des fouilleurs principaux, Hauser, n'avait jusqu'à présent pas pu être raccordé aux autres stratigraphies. Ce système correspond cependant presque parfaitement à la stratigraphie connue et documente en outre la présence de deux couches sus-jacentes (7 et 8). Le contenu de ces trois couches est étudié ici. Elles diffèrent fortement en ce qui concerne l'usure et le fractionnement des pièces, ce qui permet de les intégrer dans le système de sédimentation proposé pour La Micoque et consistant en une alternance de dépôts de versant et de dépôts fluviatiles de haute énergie. Ceci indique que l'âge des couches 6 et 7 au moins n'est probablement que légèrement inférieur à celui des couches sous-jacentes. L'étude typo-technologique des couches 6, 7 et 8 a démontré que celles-ci ne diffèrent guère l'une de l'autre, tant du point de vue du débitage, qui contient très peu de Levallois, du Discoïde, des nucléus en forme de lingot et un débitage opportuniste de courtes séries d'éclats, que du point de vue typologique. Seule la présence de nombreux objets bifaciaux dans la couche 6 la différencie clairement des deux autres. Ces outils bifaciaux obéissent à deux schémas conceptuels différents, permettant de produire soit des bifaces, soit des couteaux bifaciaux. Ces caractéristiques permettent de placer l'industrie de la couche 6 dans le Micoquien, maintenant appelé Keilmessergruppen (KMG), mais se pose la question de la pertinence d'une attribution "culturelle " sur la seule base de la présence ou de l'absence de certains éléments dits diagnostiques, en ce cas les outils bifaciaux.
\end{abstract}

Mots-clés : La Micoque, Micoquien, Keilmessergruppen, stratigraphie, unité techno-fonctionnelle, technologie, classification d'ensembles lithiques

Key-words : La Micoque, Micoquian, Keilmessergruppen, stratigraphy, techno-functional unit, technology, inventory classification.

\section{Abridged english version \\ UPPER LEVELS OF LA MICOQUE (Dordogne).}

The site of La Micoque (Dordogne, France: fig. 1) has been discovered in 1895 and excavated since 1896. Numerous researchers have been working there until Hauser rent the ground in 1907. He was accused of cooperation with the enemy and had to leave France in 1914 , the site remaining unprotected. The next excavations took place in 1929, after Peyrony had bought the site for the state, but to that time the uppermost layers were destroyed. A picture (fig. 2) shows that those layers still existed in 1912 and that their disappearance is the result of 15 years uncontrolled digging. Bordes started a small excavation in 1956 and an interdisciplinary research program using modern digging and analyse techniques began in 1983.

The stratigraphy of La Micoque presently acknowledged is based on the publication of Peyrony's 1929 excavations (Peyrony 1933, 1938). The artefacts from Hauser's excavations as well as those of Wiegers' collection, which contains numerous cores, flakes and debris, however, refer to another stratigraphical system that was regarded as incompatible with the actual one. But as Peyrony had already excavated in La Micoque in 1906, published his results and quoted that his stratigraphy was nearly identical to Hauser's (Peyrony 1908a, 1908b), it should be possible to relate Hauser's stratigraphy to the actual one. The only problem is that Peyrony doesn't make any reference to his early stratigraphy in his later publications. He was numbering the layers beginning from the lowest one, labelled $\mathrm{A}$, and was therefore forced to rename all layers if deeper ones were found during excavations. This happened in La Micoque. The first step to a correlation of Hauser's stratigraphy with the official one is thus to clear which layers of Peyrony's old stratigraphy cor- 
respond to the actual ones. This can be done relatively easily (fig. 3) by comparing the archaeological contents' description. The earlier Micoquian layer G can be identified without doubt as the layer N(6). As the content of the so-called "lower unit" (comprising the earlier layers B, C and D) is described as primitive, it corresponds to the artefacts from the later layer J. The ones from the later layers $\mathrm{I}$ and $\mathrm{K}$ are only described as rolled, but it is likely that they also belong to the former "lower unit". The concretionate basis of Peyrony's first stratigraphy, A, is hence the later layer H(4), containing a Mousterian industry which he didn't excavate in 1906. Once this settled, Hauser's stratigraphy becomes easy to match with the official one (fig. 4). His lowest archaeological horizon, $L_{\text {Hauser }}$, corresponds with the layer $\mathrm{H}(4)$ because both contain a typical Mousterian industry (Peyrony 1933, 1938 ; Wiegers et al. 1913), while K, J and $\mathrm{H}_{\text {Hauser }}$ with "primitive" industry represent the former "lower unit" of Peyrony, i.e. the layers I, J and K of the official stratigraphy. The archaeological horizon discovered at the bottom of $L$ by Bordes in 1956, 5', could be Hauser's layer $G$ while the upper part of $L$ could correspond to Hauser's layer F. Consequently, $E_{\text {Hauser }}$ is $M$ and $D_{\text {Hauser }}$ is the Micoquian layer $N(6)$, as the description of their contents match to each other. But this also means that there were two more layers above the now missing Micoquian one, which aren't described anywhere else in the literature and are destroyed as well. Luckily, the collection Wiegers contains cores, tools, flakes and debris from all those layers, allowing checking the characteristics of each industry. It is clear that those layers $C$ and $B$ Hauser don't belong to the Micoquian one, as B was a thick, reddish horizon quite clearly different from $D_{\text {Hauser }}$ (fig. 5), and as their sedimentation mechanism differ. This can be demonstrated by the comparison of the artefacts' damaging grade in the three layers $D$ (the Micoquian one), $C$ and $B$ (fig. 6). To avoid confusions between the different denominations of the layers, the Micoquian one will be, following Peyrony's system for archaeological horizons, henceforth named 6 , while the layer $\mathrm{C}_{\text {Hauser }}$ becomes 7 and $\mathrm{B}_{\text {Hauser }} 8$. It becomes clear by the look on fig. 6 that layer 6 and 8 contain much lesser rolled and broken pieces than layer 7 . Moreover, the median of the maximal piece length of layer 7 is significantly lower than the one of layers 6 and 8 if all blanks, including the broken ones, are taken into account. This varies if only complete pieces are considered. Those differences can be put in relation with the interpretation of the sedimentation mechanisms observed for the lower layers by Texier \& Bertran (1993), which involves an alternance of low-energy and high-energy deposits. In consequence, 6 and 8 are slope deposits while 7 is the result of fluviatile activity, and the age of 6 and 7 at least has to be only slightly younger than the one of the underlying layers.

The industry of the layers 6,7 and 8 considered here present interesting patterns. Layer 6 , which functioned as reference for the definition of the Micoquian, is the only one of the three layers containing a certain amount of bifacial items. The analysis of their technofunctional units or TFU (Boëda 2001) shows that two concepts are present at La Micoque. First, the handaxes (fig. 7 and 8 ) present a theoretical symmetry along the length axis. Both edges near the tip are active while the basis can be active or passive. Further TFU can be placed between point and basis. The second concept corresponds to the German Keilmesser (fig. 9 and 10). Those present no length symmetry but a back opposed to an active edge. This back can take the whole length of one side or be limited to the bottom part of it, a secondary active TFU connecting to the main active one at the tip of the tool. The basis can carry an active TFU that is then shifted towards the main active TFU. This allows linking the industry discovered in 6 to the middle European Keilmessergruppen. The question of bifacial item production in situ must remain unresolved. The presence of a few thinning flakes (fig. 11) in all levels is not sufficient to attest it, but the collecting methods of the time must be taken in account. Layer 6 is not the only one that has produced bifacial items, but only two other layers, which are only described by Hauser, contained some comparable to the ones known from layer 6: $P$ and $Q$ (fig. 12). This layers seem however not to be in stratigraphical original position and could belong to 6 .

The industry of this layer contains only one Levallois core (fig. 13,1) and few discoid ones (fig. 13, 2). The most important knapping techniques are represented on one hand by the ingot-shaped cores (fig. 14), allowing the production of thin wide flakes, and on the other hand by globular cores (fig. 15) in all states of reduction, which have produced irregular flakes and an important amount of éclats débordants (they represent $25 \%$ of all blanks in the layer). This technique is particularly suitable to reduce small or irregular nodules. The Levallois flakes are unsurprisingly seldom (fig. 16, 1-2), while the most frequent tools are various side-scrapers (fig. 17, 1, 2, 3, 4, 6, 7). Notches and denticulates as well as borers (fig. 17, 5) and some atypical end-scrapers (fig. 17, 8, 9) are present.

The industry of layer 7 differs only slightly from the former one, excluding the absence of bifacial items. The Levallois technique remains rare (fig. 18, 1) but the discoid cores become more numerous (fig. 18, 2-3) while the globular cores are the most frequent ones (fig. 19, 1-2), producing again numerous éclats débordants, and the ingot-shaped cores are less represented (fig. 19, 3). No new knapping technique could be identified. Wiegers' collection is the only one containing tools coming from this layer. One Levallois blade is present (fig. 16,3 ) and the side-scrapers are again the most numerous tools (fig. 20, 1-6). End scrapers (13) are relatively numerous (fig. 20, 7-8) while notches and denticulates are rare due to the strict sorting of rolled and damaged pieces needed for this layer. One borer and two atypical backed knifes, one with borer point, are present (fig. 20, 9-10).

The layer 8 has provided only one bifacial item (fig. 21). The number of Levallois (fig. 22) and discoid (fig. 23) cores increases slightly, but the globular cores remain most important (fig. 24, 1) and the ingot-shaped cores are still present (fig. 24, 2). The Levallois blanks (fig. 16, 4-10) are much more numerous than in the other layers. Only one tool from this layer comes from another collection than Wiegers' (fig. 25, 1), probably because beautiful big tools were rare. The side-scrapers are varied (fig. 25, 2-6), the end-scrapers relatively frequent and more typical than in the two other layers (fig. 26, 1-3). Borers are missing, but three atypical burins (fig. 26, 4-5) are present.

The three layers present striking similarities concerning the knapping methods, and their toolkits are rather comparable in the cumulative diagram. But the layer 6 is the only one containing the bifacial items typical for the Keilmessergruppen while both other industries can be best related to the Mousterian of Quina type. The question is, however, if this distinction is justified, or, in other words, if the presence or absence of bifacial items is a sufficient argument to discriminate "cultural entities". The reasons leading to the accumulation of certain types of tools in certain types of camps are too diverse and still not investigated as much as necessary to allow any conclusion. 


\section{1 - LE SITE}

La Micoque est située non loin du village des Eyzies-deTayac en Dordogne (fig.1) et domine d'environ $20 \mathrm{~m}$ (comm. pers. J.-P. Texier) le ruisseau Manaurie, affluent de la Vézère (Chauvet et Rivière 1896 ; Laville 1975). Le site doit son nom à un mas ruiné sur la propriété duquel les artéfacts furent découverts (Bordes 1984a).

Le site de La Micoque était, lors de sa découverte, une surface parsemée de blocs d'éboulis présentant une pente de $22^{\circ}$. Durant les fouilles, une falaise calcaire devant laquelle les sédiments s'étaient accumulés fut dégagée. La Micoque était vraisemblablement un site de plein air au pied d'une paroi rocheuse.

\section{2 - UN PEU D'HISTOIRE, PROBLÈMES ET QUESTIONS}

\section{1 - De nombreux chercheurs}

Le site fut découvert en 1895 et les premières fouilles furent faites en 1896 par G. Chauvet et E. Rivière, qui publièrent immédiatement les résultats (Chauvet 1896 ; Chauvet et Rivière 1896). Ceci incita de nombreuses personnes à entreprendre des fouilles à La Micoque dans les années qui suivirent, tels en 1896 Capitan (Capitan 1896), en 1897 Harlé, en 1898 et 1906 Peyrony (Peyrony 1908a et b), en 1903 et 1905 Coutil (Coutil 1905), en 1905 Cartailhac, en 1906 et 1907 Hauser (Hauser 1906-1907). A partir de 1907, Hauser loua le terrain afin d'écarter tous les autres chercheurs. En 1912, trois scientifiques berlinois se rendirent aux Eyzies et fouillèrent à La Micoque. Cette mission se révèle maintenant être d'une grande importance car la publication qui en résulta (Wiegers et al. 1913), bien que n'étant qu'un rapport préliminaire, nous donne une deuxième description de la stratigraphie d'Hauser et la confime, du moins en grande partie.

En 1914, Hauser, accusé de collaboration avec l'ennemi, dut quitter Les Eyzies en toute hâte. II n'y revint jamais. Ce n'est qu'en 1929 que Peyrony, après avoir acheté le terrain pour l'Etat, y entreprit de nouvelles fouilles qui mirent au jour une série de couches archéologiques inconnues jusque-là (Peyrony 1933, 1938). Le site étant resté quinze ans sans protection, les couches supérieures, encore présentes en 1912 (fig.2), étaient détruites lors de la reprise des travaux. Seuls quelques restes à droite du site purent encore être identifiés (Peyrony 1938). En 1956, Bordes entreprit un sondage à La Micoque (Bordes 1984a), suivi par une reconsidération de la stratigraphie par Laville (Laville 1975 ; Laville et Rigaud 1976). A partir de 1983, de nouvelles fouilles ont été entreprises par une équipe interdisciplinaire (Debénath et Rigaud 1986).

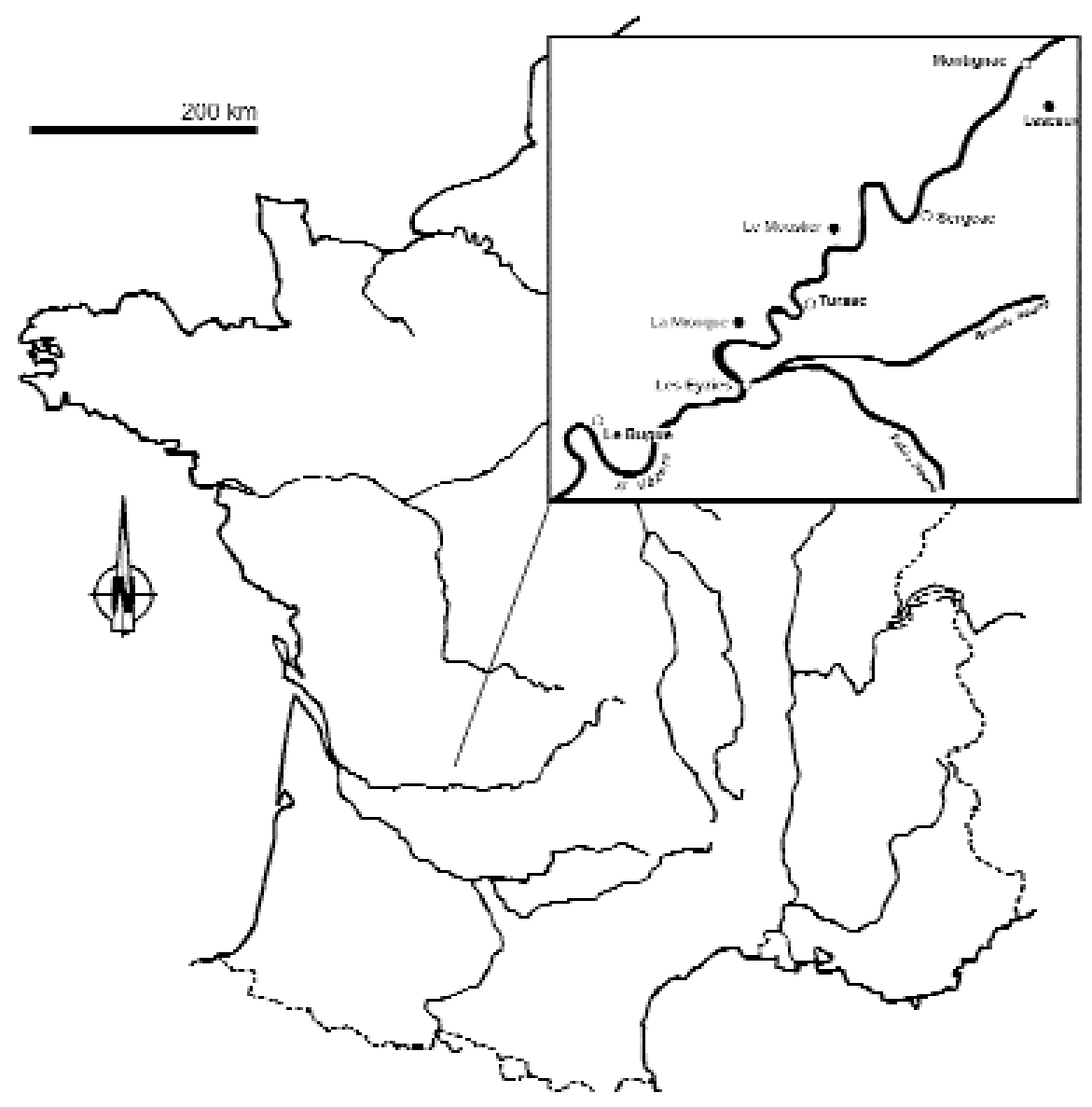

Figure 1 - situation de La Micoque.

Figure 1 - situation of La Micoque. 


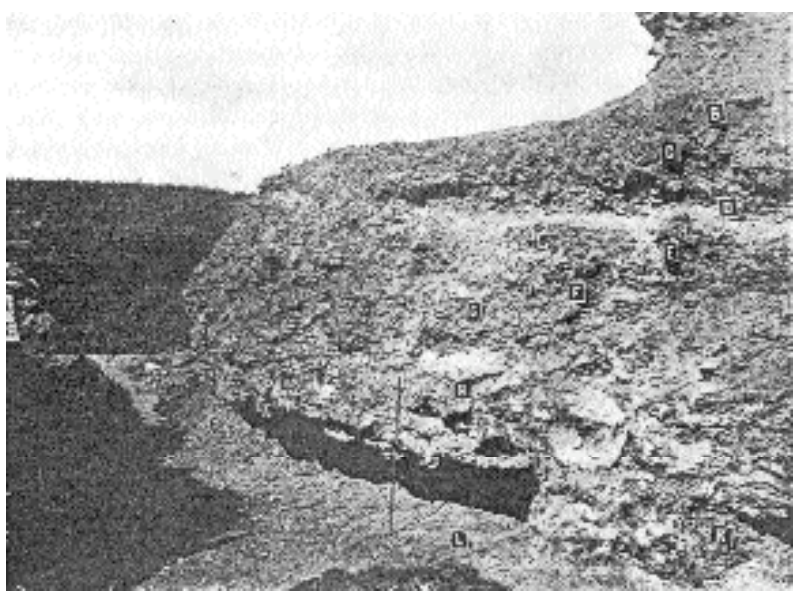

Figure 2 - photographie de la stratigraphie de La Micoque en 1912 (Wiegers et al. 1913).

Figure 2 - picture of the stratigraphy of La Micoque in 1912 (Wiegers et al. 1913).

L'histoire mouvementée du site a provoqué la dispersion des collections dans de nombreuses institutions, tant en Europe qu'en Amérique. Souvent, les pièces ne portent plus aucune indication stratigraphique.

La couche 6, qui contenait les bifaces, a disparu aujourd'hui. II s'agit de la couche éponyme du Micoquien (Bosinski 1967 ; Hauser 1916 p.55), dont plusieurs auteurs tentèrent au cours du $20^{\circ}$ siècle de reconstituer, décrire et définir le contenu (Bordes 1984b ; Bosinski 1970 ; Patte 1971). La discussion concernant le Micoquien et les industries de la Micoque se poursuit encore à l'heure actuelle (Gouédo 1999 ; Richter 1997, 2002 ; Veil et al. 1994).

\section{2 - De nombreuses stratigraphies}

La première description de la seule couche connue à l'époque parut en 1896 (Chauvet 1896 ; Chauvet et Rivière 1896). Capitan publia en 1907 un relevé des couches découvertes par Peyrony et, en 1908, Hauser présenta son interprétation de la coupe, où figure une seule couche archéologique qui suit la pente du terrain (Hauser 1908a). Après la publication par Peyrony de son interprétation de la stratigraphie (Peyrony 1908a), Hauser publia un nouveau profil consistant en une succession de couches horizontales (Hauser 1908b) qui trouva l'approbation de Peyrony (Peyrony 1908b). Les travaux de Wiegers (Wiegers et al. 1913) confirment également cette seconde stratigraphie d'Hauser.

La publication des résultats des fouilles de 1929 (Peyrony 1933, 1938) marque d'une certaine façon un nouveau commencement, puisque Peyrony ne mentionne ni ne fait référence aux stratigraphies antérieures. Cette "nouvelle " stratigraphie servit, à partir de ce moment, de base pour tous les travaux postérieurs et est encore aujourd'hui la stratigraphie de référence. Cette stratigraphie fut précisée et complétée par Laville (Laville 1975 ; Laville et Rigaud 1976) et Bordes (Bordes 1984a) puis complètement réinterprétée en 1993 (Texier et Bertran 1993).

A partir du moment où la nouvelle stratigraphie de Peyrony fut adoptée comme base de recherche, toutes les stratigraphies antérieures perdirent leur valeur et ne furent jamais mises en relation avec celle-ci. Ceci concerne autant la première stratigraphie établie par Peyrony que la stratigraphie d'Hauser, dont Peyrony assure qu'elles sont pour ainsi dire identiques (Peyrony 1908b). II en découle que tous les objets en provenance de collections faisant référence à la stratigraphie d'Hauser ne peuvent être raccordés au système stratigraphique actuel. Ceci inclut non seulement les objets vendus par Hauser, mais aussi un ensemble de plusieurs milliers de pièces en provenance de toutes les couches connues à l'époque, collecté par le géologue berlinois Wiegers en 1912 (Wiegers et al. 1913). Cette collection contient, outre un certain nombre de pièces retouchées, de nombreux nucléus et déchets de débitage, permettant d'ébaucher une étude technologique de la couche 6 . Au vu des questions non résolues concemant le contenu de la couche 6 , il est donc impératif de tenter de corréler la stratigraphie d'Hauser avec la seconde stratigraphie de Peyrony.

\section{3 - CORRÉLATIONS}

Hauser ne modifia que très peu la stratigraphie qu'il publia en 1908 et dont Peyrony dit qu'elle correspond exactement à la sienne (Peyrony 1908b). Ceci signifie qu'il ne découvrit pas de couches archéologiques supplémentaires telles celles que Peyrony découvrit en 1929 et que la base de la première stratigraphie de Peyrony correspond à peu près à celle de la stratigraphie d'Hauser. Dès lors, si la première stratigraphie de Peyrony peut être corrélée avec celle des fouilles de 1929, la base de la stratigraphie d'Hauser pourra être mise en relation avec une couche de la stratigraphie de Peyrony 1933. La comparaison des descriptions des couches permettra ensuite d'évaluer dans quelle mesure les couches d'Hauser correspondent aux couches connues actuellement. Les descriptions des couches et les coupes stratigraphiques pouvant être consultées dans les diverses publications, elles ne seront reprises ici qu'en cas de besoin.

\section{1 - Les stratigraphies de Peyrony (Capitan 1907 ; Peyrony 1908a, 1933, 1938)}

Peyrony avait pris l'habitude de numéroter ses couches de bas en haut, s'obligeant ainsi à modifier l'appellation de toutes les entités chaque fois que des couches plus profondes faisaient leur apparition. Ce fut le cas à La Micoque, où les couches identifiées lors des fouilles de 1906 portent d'autres appellations que celles des fouilles de 1929. Une seule fois, Peyrony fait référence à son ancienne stratigraphie dans une publication tardive : il mentionne que la couche $\mathrm{E}$ est le niveau archéologique puissant qu'il avait atteint en 1906 (Peyrony 1933).

La confrontation des informations lithologiques et archéologiques livrées par les deux descriptions stratigraphiques (fig. 3) permet immédiatement de se rendre compte que Peyrony n'a jamais atteint la couche E dans ses fouilles de 1906. En effet, même si toutes les couches ne peuvent être parallélisées avec certitude, la présence de divers niveaux archéologiques permet de faire un certain nombre de rapprochements. La couche $\mathrm{G}_{\text {ancienne }}$ comespond avec cert itude à la couche $\mathrm{N}(6)$, il s'agit de la couche dite micoquienne. Etant donné que le niveau archéologique 5' à la base de L n'a été reconnu que bien plus tard par Bordes 
Premiere stratigraphie de Peyrony

(Peyrony 1908)

$\mathrm{H}$ : dépóts de pente
Deuxiérne stratigraphie de Peyrony (Peyrony 1933,1938, Laville 1978

O: dépóts de pente

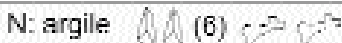

$\mathrm{M}$ : conglomćral

L: meuble

(1) $15\left(5^{\prime}\right)$

K: congloméral

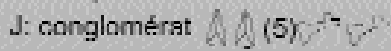

I: conglomérat

$\mathrm{H}$ : suble meuble $6(4) \mathrm{G}$

G: corilgumiral sableux

F: éboulis \& 4

E

D

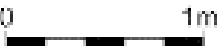

Figure 3 - La Micoque : corrélation des stratigraphies de Peyrony.

Figure 3 - La Micoque : correlation of the stratigraphies of Peyrony. 


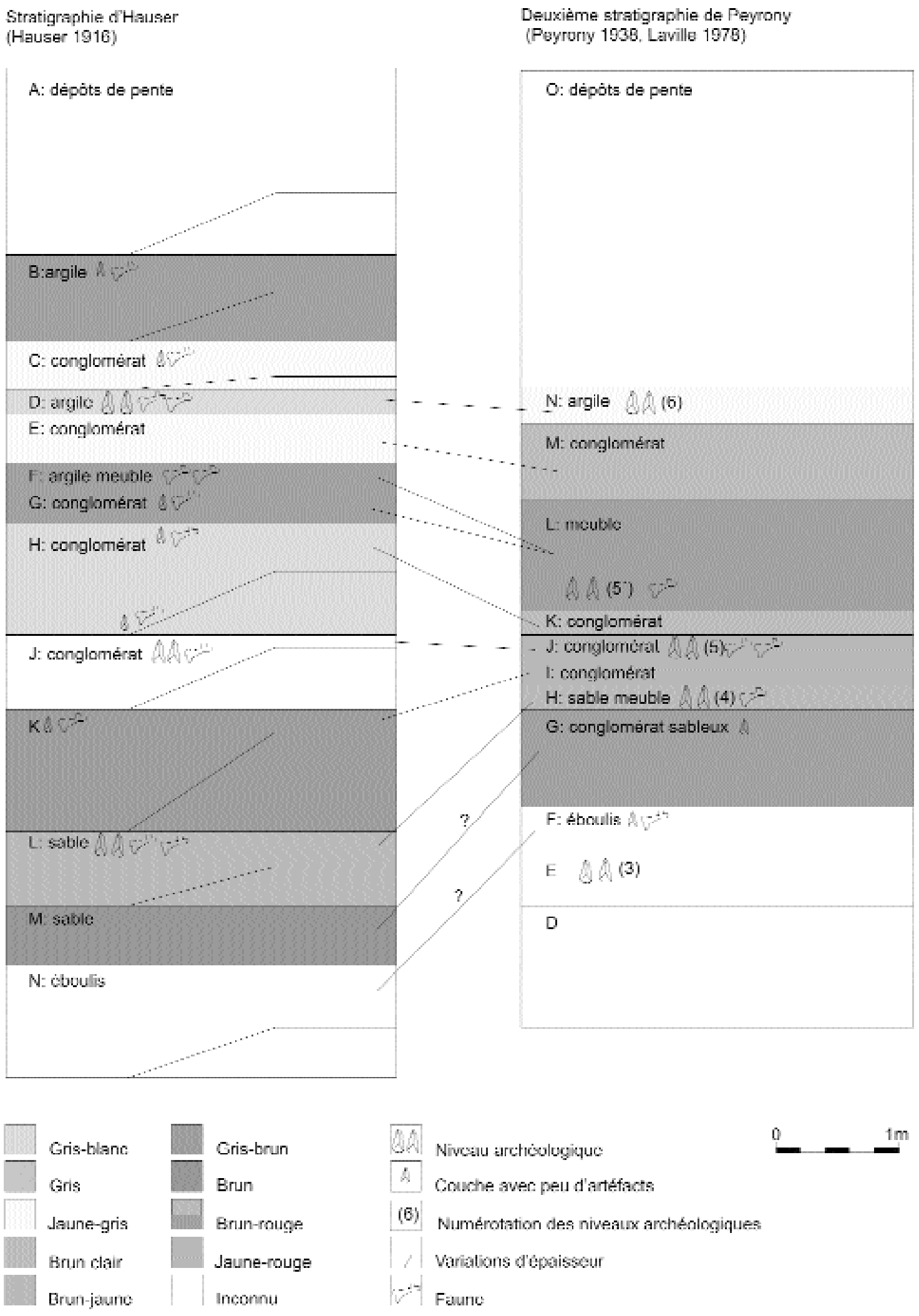

Figure 4 - La Micoque : corrélation de la stratigraphie d'Hauser avec la stratigraphie de référence.

Figure 4 - La Micoque : correlation of the stratigraphy of Hauser with the official one. 
(1984a), il n'a probablement pas été identifié en 1906 par Peyrony et ne peut être pris en compte. Le groupe de trois couches $\left(B, C, D_{\text {anciennes }}\right)$, contenant du matériel archéologique et considéré (Peyrony 1908a) comme le niveau inférieur de La Micoque, correspond très probablement aux couches $\mathrm{K}, \mathrm{J}(5)$ et peut-être I. Cette hypothèse est confirmée par la description du matériel archéologique recueilli dans ces couches. Les pièces provenant de $B, C$ et $D_{\text {anciennes }}$ sont décrites comme grossières, épaisses et infomes (Peyrony 1908a), celles provenant de la couche $\mathrm{J}(5)$ également (Peyrony 1938). La seule précision foumie concernant le contenu des couches $\mathrm{K}$ et $\mathrm{I}$ est que les pièces sont roulées. II est fortement improbable que Peyrony ait atteint la couche $\mathrm{H}(4)$ en 1906, les artéfacts en p rovenant étant décrits comme appartenant au Moustérien classique, avec des éclats assez minces, du débitage discoïde, et presque tous les types d'outils du Moustérien typique (Peyrony 1938), genre d'industrie qui n'est pas décrit dans le contenu de l'entité inférieure des fouilles de 1906 (Peyrony 1908a). La base du profil de 1906 , la couche $A_{\text {ancienne, }}$, non fouillée, pourrait donc correspondre à la couche $\mathrm{I}$, ou plus probablement à la couche $H(4)$, bien que celle-ci ne soit pas cimentée dans le profil de 1929, cette cimentation étant un phénomène postdépositionnel local (Texier in Debénath et al. 1991).

II est intéressant de noter que l'outillage recueilli dans la couche $\mathrm{J}(5)$ est décrit par Peyrony (1938) comme ayant " beaucoup plus d'affinité avec celui du niveau E qu'avec celui de la couche $\mathrm{H}$ ", ce qui pourrait expliquer pourquoi Peyrony a confondu $\mathrm{E}$ avec son niveau inférieur des fouilles de 1906.

Les différences entre les deux profils ne peuvent être expliquées que s'ils n'ont pas été relevés au même endroit. II n'existe malheureusement aucune documentation nous permettant de déterminer où ces profils se trouvaient, ni à quelle distance ils étaient l'un de l'autre. II n'existe aucun relevé longitudinal de la stratigraphie de La Micoque, les fouilles d'Hauser ayant détruit une grande partie du profil le long de la falaise.

3.2 - Hauser et la stratigraphie de référence (fig. 4) Dans son article de 1908, Peyrony précise également que les couches $B, C$ et $D$ Hauser correspondent à son entité supérieure, et que les couches $\mathrm{J}, \mathrm{K}$ et $\mathrm{L}_{\text {Hauser }}$ comespondent à son entité inférieure, composée de $B, C$ et $D$ (Peyrony 1908b). Ces entités sont séparées chez Peyrony par une zone stérile.

Cette mise en parallèle n'est pas absolument exacte. Peyrony, qui ne put fouiller à La Micoque que jusqu'en 1906, dut rester à la surface de la brèche formant la base de sa stratigraphie. Hauser quant à lui, semble, dès 1908 , avoir fouillé dans une partie du gisement où cette couche, comespondant à la couche $\mathrm{H}(4)$, n'était pas cimentée par les carbonates. En effet, Wiegers et ses collaborateurs (1913) signalent que les racloirs moustériens typiques dominent dans la couche $L_{\text {Hauser, }}$, ce qui correspond à la description du contenu de la couche archéologique $\mathrm{H}(4)$ (Peyrony 1933). D'un point de vue archéologique, il est plus que probable que la couche $\mathrm{J}_{\text {Hauser }}$, contenant de très nombreux petits silex roulés donnant à l'inventaire un caractère primitif, correspond à la couche $\mathrm{J}(5)$ à industrie grossière, les couches $\mathrm{H}$ et $\mathrm{K}_{\text {Hauser }}$ comespondant dès lors aux couches $\mathrm{K}$ et $\mathrm{I}$. Ceci permet de fixer la base de la stratigraphie d'Hauser par rapport aux couches connues, chose confirmée dans la stratigraphie de 1916 par la présence de la couche $M_{\text {Hauser }}$ presque stérile sous la couche

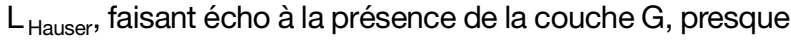
stérile, sous la couche $H(4)$. C'est probablement cette couche presque stérile reposant sur un éboulis stérile de l'avis d'Hauser mais contenant des artéfacts fortement roulés et altérés ( $\mathrm{N}_{\text {Hauser }}$ ou $\mathrm{F}$ ) qui incita Hauser à ne pas fouiller plus bas.

La " zone stérile" dominant l'entité inférieure décrite par Peyrony s'est avérée contenir une couche archéologique (5') à la base de $L$ (Bordes 1984a), qui pourrait correspondre à $G_{\text {Hauser, }}$ tandis que $F_{\text {Hauser }}$ pourrait former la partie supérieure de $L$. La couche stérile $E_{\text {Hauser }}$ se laisse corréler sans problèmes avec la couche stérile $\mathrm{M}$. Ceci nous mène à la couche $D_{\text {Hauser }}$ ou $N(6)$, la couche " micoquienne ". Son contenu est décrit de façon unanime par tous les fouilleurs, qui insistent sur la présence de bifaces finement taillés, d'une très belle industrie et d'un nombre impressionnant d'ossements et de dents de chevaux. En outre, la couche D d'Hauser, tout comme la couche $N$ (6) de Peyrony, est sub-horizontale et repose en conformité sur les couches sous-jacentes. Une telle disposition suggère que la couche $D$ appartient au même système sédimentaire que le reste de la séquence. Tous ces éléments pris ensemble permettent de postuler que la couche D Hauser comespond à la couche $N(6)$.

II n'en reste pas moins que les deux couches supérieures décrites par Hauser ne sont présentes dans aucune stratigraphie de Peyrony, qui ne semble pas s'en émouvoir puisqu'il met en parallèle son entité supérieure avec $B, C$ et $D$ Hauser (Peyrony 1908b). Mais l'entité supérieure de Peyrony se limitant à la couche $\mathrm{N}(6)$, cela signifie-t-il que les trois couches $\mathrm{B}, \mathrm{C}$ et $\mathrm{D}_{\text {Hauser }}$ comespondent toutes à la couche $\mathrm{N}(6)$ ? Outre le fait qu'aucun fouilleur ne mentionne la pré-

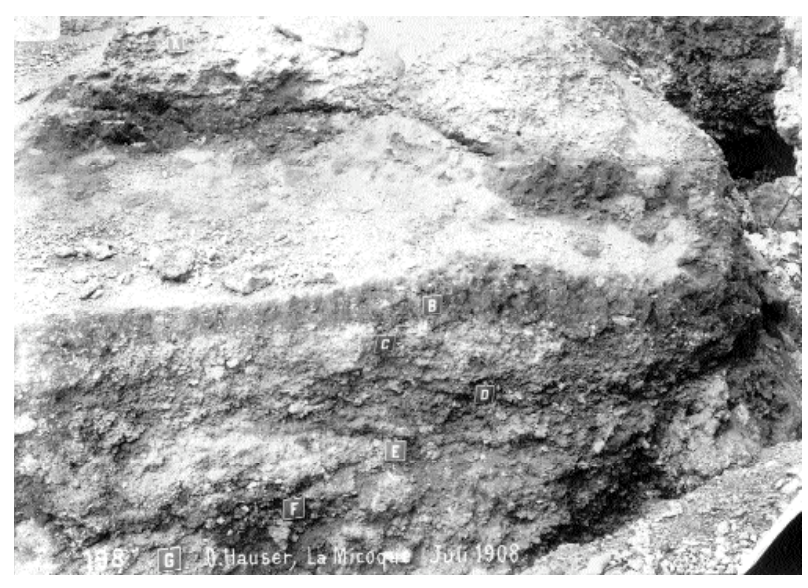

Figure 5 - photographie des couches supérieures de La Micoque faite par Hauser en $1908(D=$ couche 6, $C=$ couche $7, B=$ couche 8 ).

Figure 5 - picture of the upper layers of La Micoque made by Hauser in 1908 ( $D=$ layer 6, C= layer 7, B= layer 8$)$. 
sence d'une subdivision à l'intérieur de la couche N(6), la description de la couche $C_{\text {Hauser }}$ (Hauser 1916), au faciès comparable à celui des couches $J_{\text {Hauser }}(J)$ et $E$ Hauser $(M)$ et contenant de nombreux petits silex roulés, va à l'encontre de cette hypothèse. La couche $B_{\text {Hauser, quant à elle, ne }}$ contenait que peu de matériel archéologique et paléontologique (Hauser 1916). En outre, une photographie d'Hauser montre que la couche $B$ se détache clairement du reste de la stratigraphie (fig. 5). II semblerait donc que la couche N(6) ait été recouverte par deux niveaux archéologiques inconnus, peut-être présents dans une partie du gisement seulement. La présence dans la collection Wiegers de caisses contenant du matériel en provenance des trois couches concernées permet de tester si celles-ci doivent être considérées comme une entité stratigraphique ou non. Afin d'éviter des confusions dans la dénomination des couches, la couche $D_{\text {Hauser }}$ sera nommée à titre provisoire 6 , faisant référence à la numérotation des niveaux archéologiques par Peyrony, la couche $C_{\text {Hauser }}$ sera nommée 7 et $B_{\text {Hauser }} 8$.

\section{4 - SÉDIMENTATION}

Les processus sédimentaires de La Micoque consistent majoritairement en une succession de dépôts de pente et de dépôts fluviatiles interstratifiés (Texier in Debénath et al. 1991 ; Texier et Bertran 1993). Les sédiments fouillés par Hauser appartiennent tous à la partie supérieure du complexe F4-DP3 et probablement à DP4, c'est à dire que les couches peu remaniées des dépôts de pentes mis en place par des mécanismes apparentés à la solifluxion altement avec des dépôts fluviatiles fortement remaniés contenant des pièces concassées.

La comparaison de l'importance du nombre de pièces brisées ou roulées dans les couches 6,7 et 8 fait apparaître des différences importantes (fig. 6). Les couches 6 et 8 contiennent nettement moins de pièces brisées et surtout roulées que la couche 7 . La médiane de la longueur maximale des produits de débitage (en raison de la présence d'une répartition asymétrique, il est impossible d'utiliser les valeurs moyennes) met également en évidence à quel point le concassage d'un ensemble archéologique peut modifier son aspect général.

Au vu de ces différences, il est très probable que la couche 7 ait été mise en place sous un régime fluviatile tandis que les couches 6 et 8 sont des dépôts de pente. Ceci a pour conséquence que ces trois couches forment des entités stratigra- phiques distinctes et qu'elles s'intègrent dans le système de sédimentation mis en évidence pour les couches sousjacentes. Dès lors, seule la couche $D_{\text {Hauser }}$ correspond au sixième niveau archéologique " micoquien", tandis que les couches $C$ et $B_{\text {Hauserr }}$ représentent des entités indépendantes dont la dénomination (7 et 8) est justifiée. Ceci signifie que le niveau 6 n'est pas en position remaniée, et que, s'il est possible de le retrouver sur le site, les sédiments pourraient éventuellement être datés. Les différents arguments stratigraphiques indiquent que la couche 6 fait partie du système sédimentaire ayant mené á l'accumulation des niveaux appartenant aux complexes DP3 et F4. Etant donné que la couche 6 recouvre les couches sous-jacentes sans les éroder, il est également peu probable qu'un hiatus important ne la sépare de celles-ci. La couche 6 n'est donc probablement que légèrement plus récente que les couches $E$ à $M$, datées des stades isotopiques 11 à 8 (Falguères et al. 1997 ; Schwarcz et Grün 1988). Le niveau archéologique découvert à la base de DP4 et remanié durant l'Holocène (Texier et Bertran 1993) ne peut donc être la couche 6 , recouverte par un niveau fluviatile, mais il est possible qu'il corresponde à la couche 8 . Le contenu du niveau archéologique à la base de DP4, ayant livré du débitage et des outils mais pas de bifaces, pourrait éventuellement être apparenté à la couche dite micoquienne selon Debénath et al. (1991). II est cependant possible que la couche 8 contienne une industrie comparable en bien des points à celle de la couche 6 , les rendant indiscernables sur la base d'un échantillon réduit. Seule une comparaison des caractéristiques technologiques et typologiques des couches 6 et 8 permettra de voir si celles-ci peuvent être différenciées uniquement à l'aide de leur débitage et de leurs outils non bifaciaux.

Dans la description des industries, seul le contenu des couches supérieures 6,7 et 8 , moins connues, sera présenté.

\section{5 - L'INDUSTRIE DE LA COUCHE 6}

\section{1 - Outils à retouche bifaciale}

Les " bifaces de La Micoque " ont déjà été décrits à de nombreuses reprises (Bordes 1961 ; 1984b ; Bosinski 1970 ; Hauser 1916 ; Obermaier 1908 ; Patte 1971), mais les discussions en cours concernant le Micoquien, sa définition, sa chronologie, son étendue géographique et ses pièces diagnostiques exigent une réévaluation des caractéristiques technologiques de ces pièces. Dans le cadre d'une redéfinition du Micoquien d'Europe centrale

\begin{tabular}{|c|r|r|r|r|}
\hline Couche & pièces hrisés & pièces roules & $\mathrm{I}_{\text {max }}$ loules pièces & $\mathrm{I}_{\text {max }}$ piéces entières \\
\hline 6 & $26 \%$ & $4 \%$ & $43 \mathrm{~mm}$ & $44,5 \mathrm{~mm}$ \\
\hline 7 & $\mathbf{4 6} \%$ & $\mathbf{3 0} \%$ & $\mathbf{3 6} \mathrm{mm}$ & $42 \mathrm{~mm}$ \\
\hline 8 & $33 \%$ & $3 \%$ & $50 \mathrm{~mm}$ & $51 \mathrm{~mm}$ \\
\hline
\end{tabular}

Figure 6 - La Micoque : comparaison des degrés de roulage et de concassage ainsi que des médians des longueurs maximales des produits de débitage avec et sans pièces brisées pour les couches 6, 7 et 8.

Figure 6 - La Micoque: comparison of the damaging degrees and the median of the maximal length of the blanks with an without broken pieces for the layers 6, 7 and 8. 

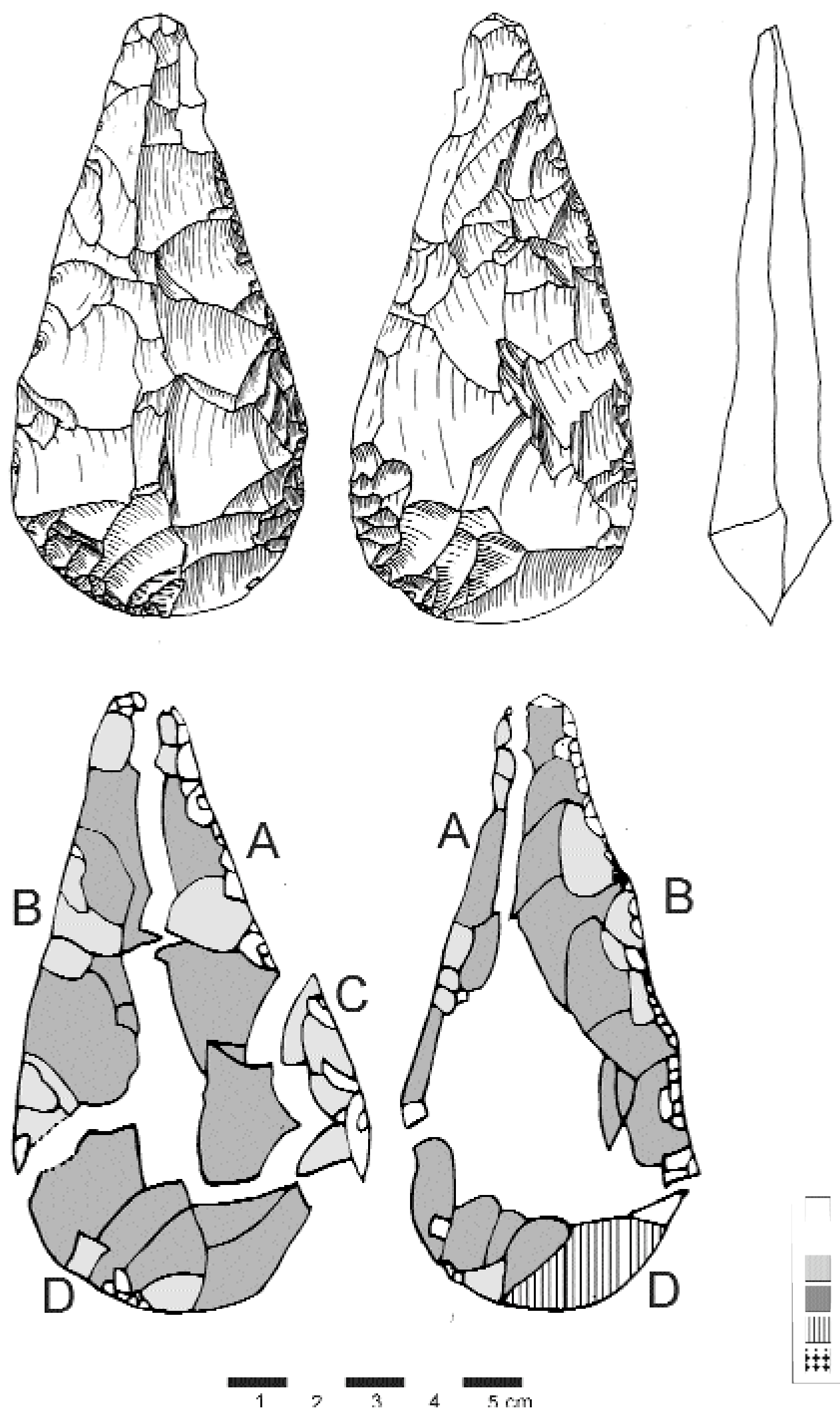

Retouche margınale / traces d'utilisation Retouche couvrante Armıncıssement Mise en forme $¥$ Anclenne surface

Figure 7 - La Micoque, couche 6 : biface avec base active/passive et UTF rajoutée en cours d'utilisation (C, passive).

Figure 7 - La Micoque, layer 6: biface with active/passive basis and a TFU (techno-functional unit) added during use (C, passive). 

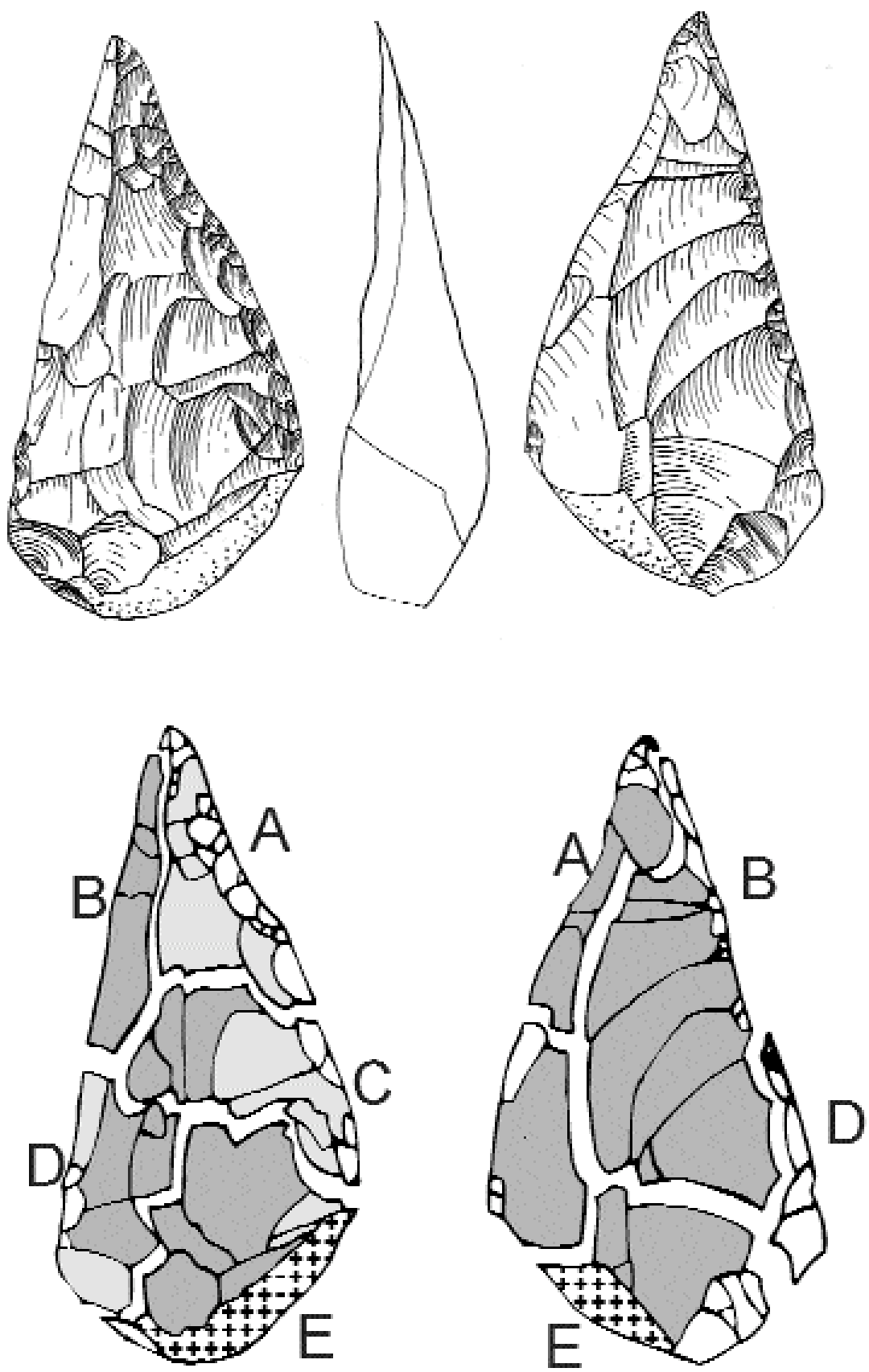

Figure 8 - La Micoque, couche 6 : biface dont les UTF $C$, $D$ et $E$ sont passives.

Figure 8 - La Micoque, layer 6: biface which TFU C, $D$ and $E$ are passive.
Retouche marginale / traces d'utilisation

Retouche couvrante Arnıncıssement Mise en forme Anclenne surface

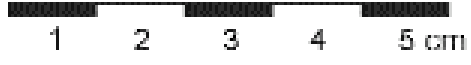

(Jöris 2001 ; Richter 1997 ; Veil et al. 1994), celui-ci s'est transformé en une entité appelée " groupes à couteaux bifaciaux " (Keilmesserg ruppen ou KMG), caractérisée principalement par la présence d'outils bifaciaux opposant un dos à un tranchant actif. Comme le pluriel du nom l'indique, cette entité présente une grande variabilité, et ses limites, tant chronologiques que géographiques, sont encore floues. Les grandes ressemblances unissant les ensembles lithiques de La Micoque 6 et de Bockstein Illb (Bosinski 1967), tous deux non datés, justifient une analyse des concepts présents à La Micoque 6. Cette analyse est faite à l'aide du concept des unités techno-fonctionnelles (Boëda 2001), basé sur l'identification d'unités de fabrication distinctes remplissant des fonctions particulières et présentant des caractéristiques morphologiques mises en place par des séries d'enlèvements indépendantes les unes des autres. Une unité technofonctionnelle (UTF) peut être active, passive ou les deux tour à tour, faire partie du concept volumétrique initial de l'outil ou avoir été ajoutée par la suite. 

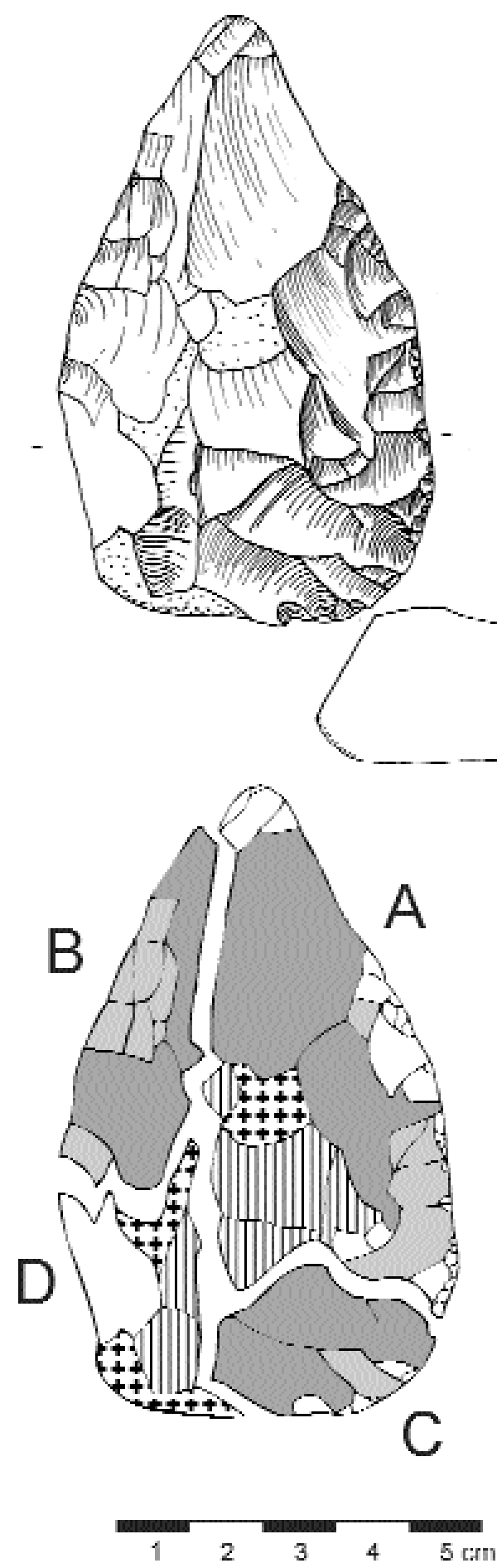

Figure 9 - La Micoque, couche 6 : Keilmesser avec tranchant principal $(A)$, secondaire (D) et base (C) actifs. $D=$ dos.

Figure 9 - La Micoque, layer 6: Keilmesser with active main cutting edge (A), secondary cutting edge (D) and basis (C). $D=$ back.
Retouche margınale / traces d'utilisation

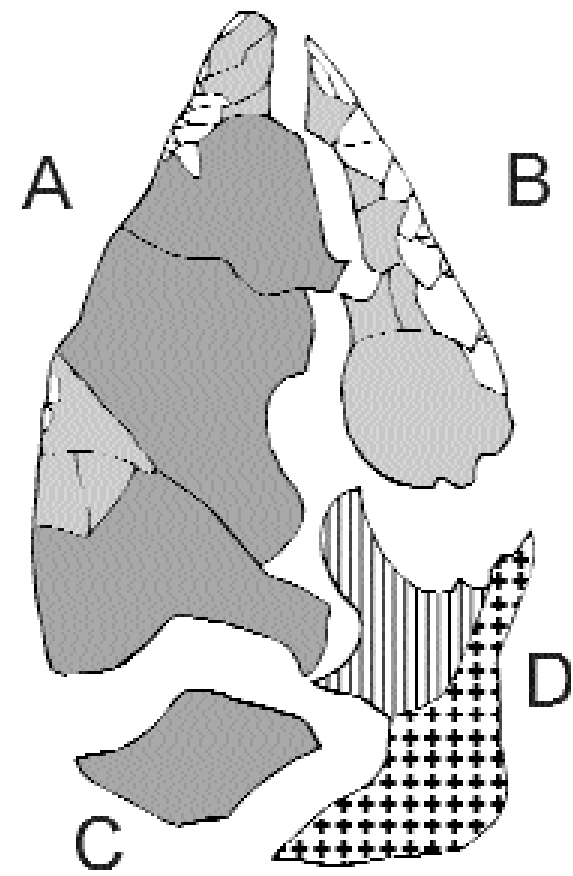

Retouche couvrante Amıncıssement Mise en forme A Anclenne surface
5.1.1- Types d'outils

Deux concepts sont présents dans la couche 6 de La Micoque. Le premier, le plus important, correspond aux bifaces et est caractérisé par une organisation relativement symétrique le long de l'axe de l'outil (fig. 7 et 8 ). La base est généralement passive mais peut également être active, les deux tranchants de part et d'autre de la pointe sont actifs. D'autres unités, actives ou passives, peuvent être présentes entre la base et la pointe. Cette symétrie est conceptuelle et ne se traduit pas forcément en une symétrie formelle de l'outil. Ces outils, qui peuvent être classés selon les critères typologiques classiques, ont pu être utilisés en tout cas d'au moins deux façons, car ils peuvent être tenus dans la main d'au moins deux façons différentes (Boëda 2001). Le second concept, nettement plus rare, comespond aux Keilmesser allemands et ne présente pas de symétrie axiale (fig. 9 et 10). Ces pièces sont caractérisées par un tranchant principal actif situé sur l'un des 

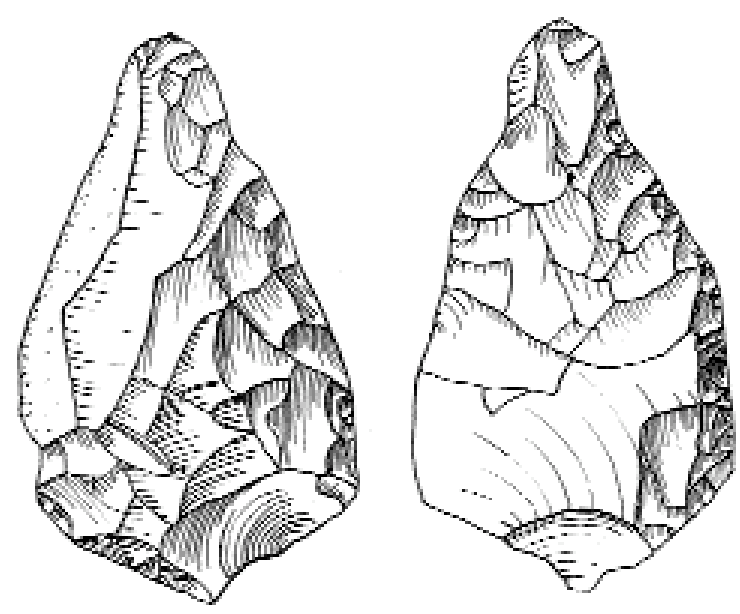

Figure 10 - La Micoque, couche 6 : Keilmesser. Le dos occupe toute la longueur du côté opposé au tranchant actif. La base (B) est peut-être active.

Figure 10 - La Micoque, layer 6: Keilmesser. The back covers the whole length of the edge opposite to the active one. The basis $(B)$ is perhaps active.
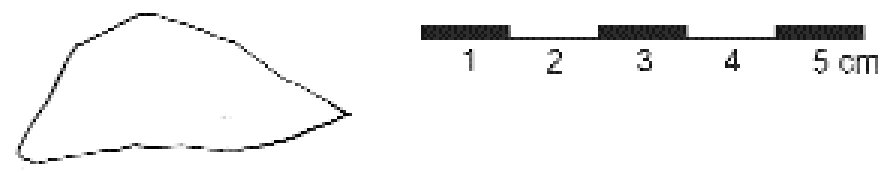
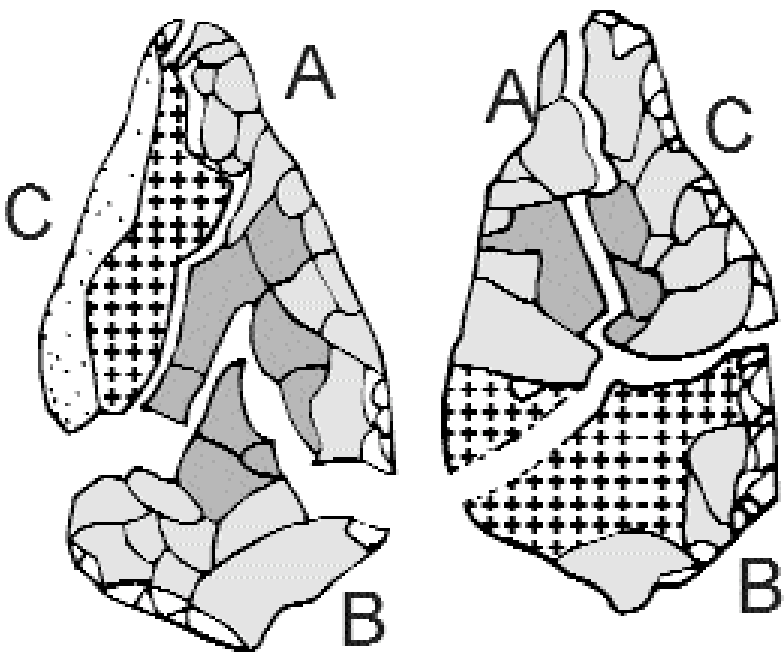

Retouche margınale / traces d'utilisation

Retouche couvrante Amıncıssement Mise en forme i\#⿱ Anclenne surface côtés et opposé à un dos passif. Un tranchant secondaire peut se situer entre la pointe et le dos et former une sorte de prolongement du tranchant principal, dont il n'atteint cependant jamais les dimensions. II est utilisé en même temps que le tranchant principal et l'outil ne doit pas être tourné dans la main pour l'activer. La base peut être active, et dans ce cas, l'unité techno-fonctionnelle qui la forme est décalée en direction du tranchant actif. Le couteau doit cependant être retoumé dans la main pour pouvoir utiliser la base en tant qu'outil. Les outils bifaciaux de La Micoque répondant au concept Keilmesser peuvent rarement être classés selon les types mis en évidence en Allemagne. Ils ressemblent souvent à des bifaces normaux mais présentent un concept différent. Toutes les pièces présentent de nombreuses traces de ravivage des bords mais aucune trace de réorganisation volumétrique ni de reprise importante, indiquant que leur biographie fut relativement courte. II est intéressant de remarquer que la caractéristique volumétrique " biface à face plane " est présente dans les deux concepts.

\subsection{2- Production in situ?}

Dans l'inventaire de la couche 6 , seuls trois éclats de façonnage ont pu être identifiés (fig. 11, 1-2). Six pièces proviennent de la couche 7 (fig. 11, 5-7) et trois de la couche 8 (fig. 11, 3-4). Tous les éclats provenant de la couche 7 ont un talon lisse, indiquant une retouche couvrante d'outils sur éclat. II est surtout intéressant dans ce contexte de remarquer la présence de six éclats de façonnage dans la couche 7 , la plus touchée par la fragmenta- 

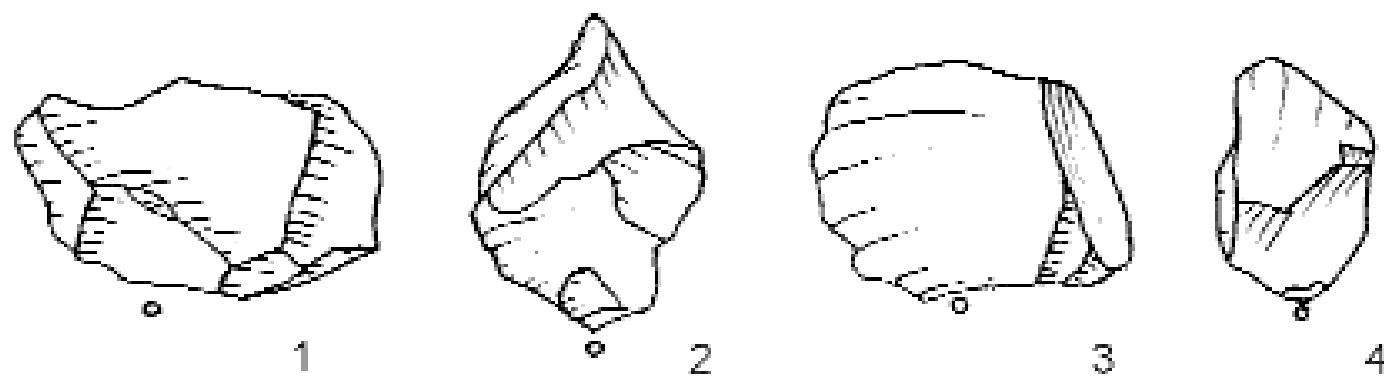

3

4
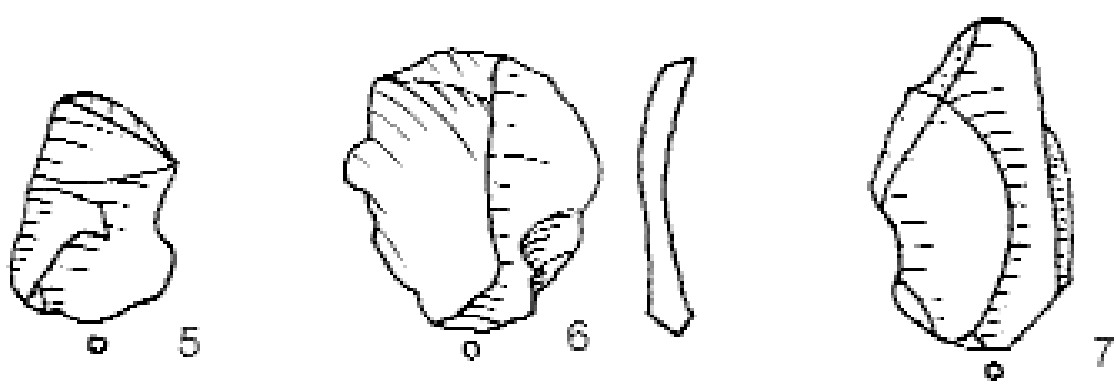

Figure 11 - La Micoque, éclats de façonnage des couches 6 (1-2), 7 (5-7) et 8 (3-4).

Figure 11 - La Micoque, thinning flakes from layer 6 (1-2), 7 (5-7) and 8 (3-4).

tion et n'ayant livré aucune pièce bifaciale. Ces éclats de façonnage sont nettement plus nombreux que dans les couches 6 et 8 , moins fragmentées et contenant des pièces bifaciales. La taille maximale moyenne de tous les produits de débitage de la couche 7 est nettement moins élevée que dans les autres couches, ce qui a apparemment incité les fouilleurs à collecter davantage de pièces de petite taille. II est donc impossible de décider si l'absence de déchets de façonnage est due aux techniques de fouille ou à l'importation de bifaces finis sur le site. La présence d'une ébauche de biface semble cependant indiquer qu'au moins une partie des outils bifaciaux a été produite sur place.

\subsection{3- Autres bifaces à La Micoque}

La couche 6 n'est pas la seule à avoir livré des bifaces. En fait, presque toutes les couches de La Micoque en contiennent (Bosinski 1970), mais ils sont nettement moins nombreux et ne présentent pas les caractéristiques typiques des pièces bifaciales de la couche 6. II existe cependant deux couches faisant exception. Ces couches, appelées par Hauser $P$ et $Q$, contenaient en grand nombre des pièces bifaciales identiques à celles de la couche 6 . Là aussi, les deux concepts de fabrication ainsi que toutes les caractéristiques métriques, typologiques et technologiques des pièces de la couche 6 sont présents (fig. 12). Sans indications stratigraphiques, ces pièces sont indiscemables les unes des autres. Ces couches se trouvaient en position basse directement devant la falaise formant le fond du gisement. Hauser (1916) utilise cette position pour prétendre que toutes les couches sont contemporaines, puisque des sédiments contenant de belles pièces se trouvent à la même altitude que ceux contenant une indus- trie primitive. Cette façon de voir les choses a provoqué les protestations véhémentes de divers collègues (Birkner 1918 ; Peyrony 1908b) qui reconnaissent clairement dans le profil d'Hauser un éboulis ayant perturbé les couches à l'arrière du gisement. Dans l'état actuel des connaissances, il est probable que Hauser, le seul à avoir fouillé à l'arrière du gisement, s'est trouvé dans le lit du paléoManaurie (Turq, comm. pers.) sans s'en rendre compte. Cette partie du site est malheureusement détruite.

\section{2 - Nucléus}

Dans la collection Wiegers, 48 nucléus proviennent de la couche 6. Les nucléus Levallois (Boëda 1993 ; Genesteet al. 1990) ne sont représentés que par un seul exemplaire de type préférentiel (fig. 13, 1) les nucléus discoïdes (Boëda 1993, 1995 ; Bordes 1961) par un seul nucléus mal conservé (fig. 13, 2). II n'existe aucun nucléus de type Quina (Turq 1989 ; Bourguignon 1997) ni acheuléen (Turq 2000) et les nucléus à lames présents dans le Micoquien du Bassin Parisien (Gouédo 1999) font également défaut. Le type de nucléus le plus fréquent dans cette couche (16 pièces) est le " nucléus en forme de lingot " (Luttropp et Bosinski 1971, p.44-46). Ces nucléus (fig. 14) sont en général de forme allongée et caractérisés par une surface de débitage plus ou moins plane, pouvant être corticale ou formée par la face ventrale d'un éclat, opposée à une face inférieure bombée ou de forme carénée. Le sens du débitage est perpendiculaire à l'axe d'allongement du nucléus, le débitage unipolaire à partir de l'un ou des deux longs côtés. Parfois, l'un ou l'autre éclat peut être débité perpendiculairement à partir de l'un des petits côtés. Le débitage a lieu en courtes séries de négatifs parallèles pouvant dans certains cas consister en un seul éclat. Les séries ne 

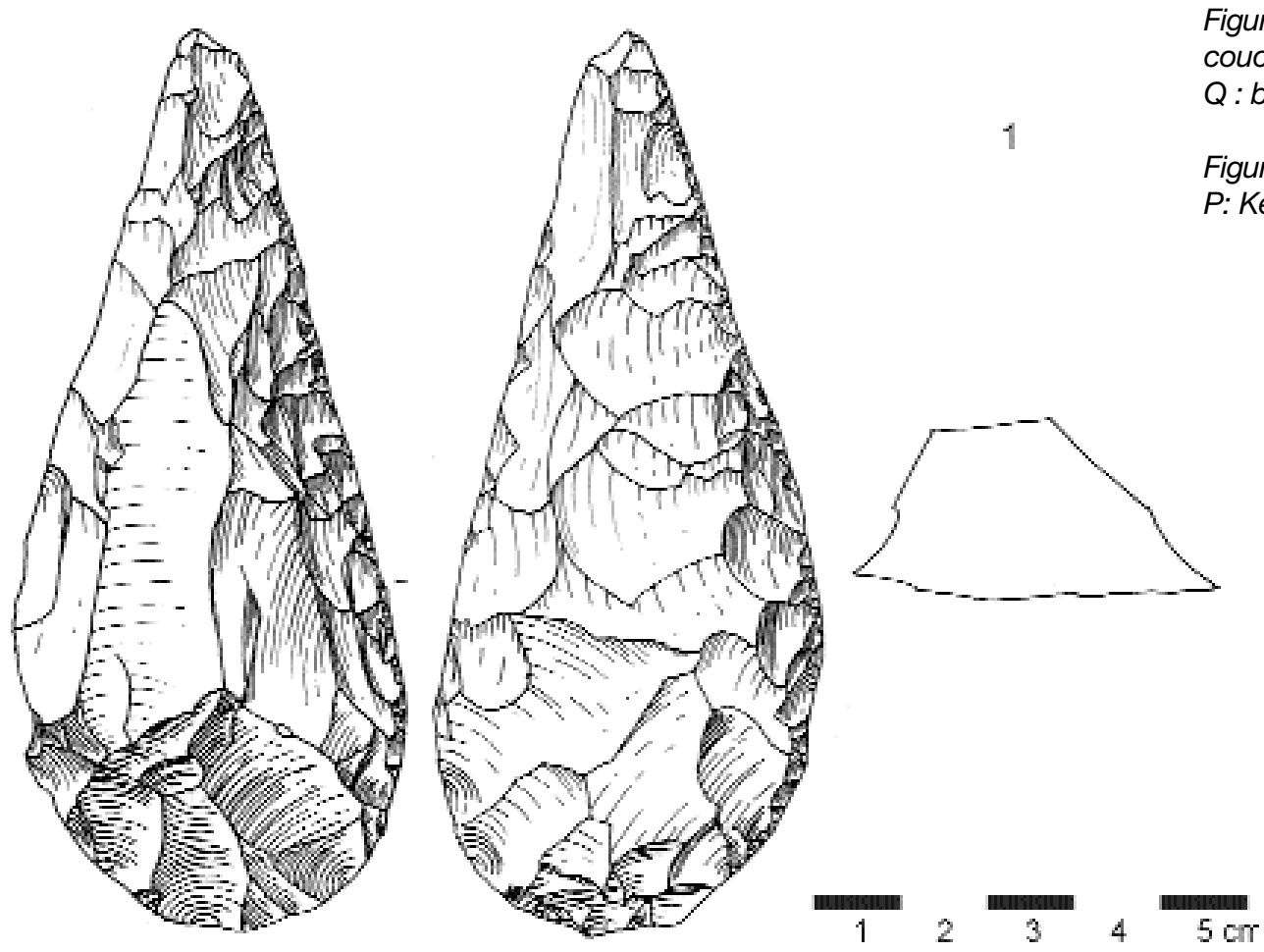
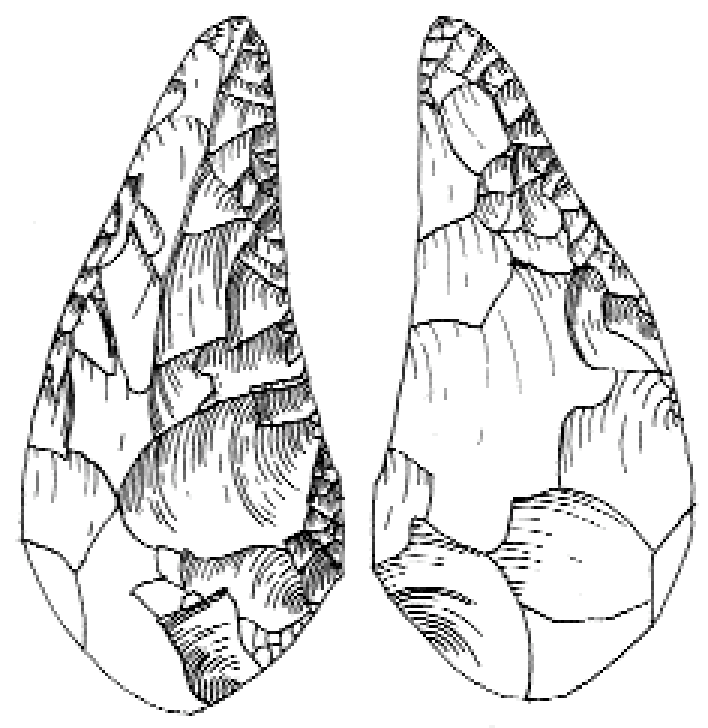

se superposent que très rarement, le nucléus étant abandonné régulièrement après le débitage d'une série sur chaque long côté. La face inférieure est souvent corticale et porte les traces d'un débitage antérieur ou d'une préparation de plan de frappe. Sur cette face, le sens du débitage varie fortement. Ces nucléus ne correspondent pas aux nucléus de type Quina définis par Bourguignon (1997), car le débitage est perpendiculaire à l'allongement du nucléus, ne touche qu'une seule surface sans changement de rôle et s'étale aux deux longs côtés, parfois même aux deux petits côtés, tandis que les convexités latérales et distales
Figure 12 - La Micoque, 1

couche $P$ : Keilmesser, 2 couche $Q$ : biface.

Figure 12 - La Micoque, 1 layer : Keilmesser, 2 layer Q: biface. 

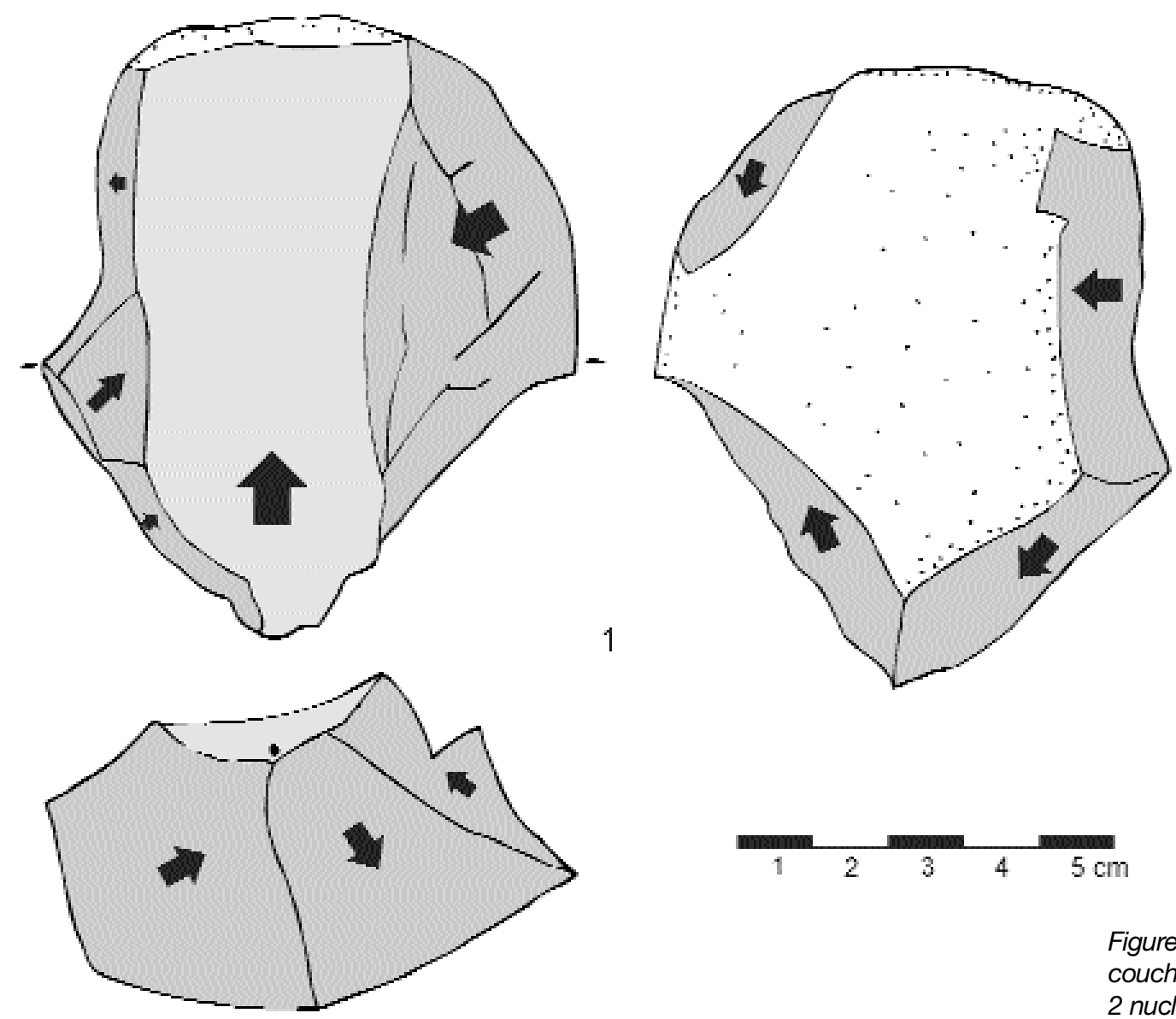

Figure 13 - La Micoque, couche $6: 1$ nucléus Levallois, 2 nucléus discoïde.

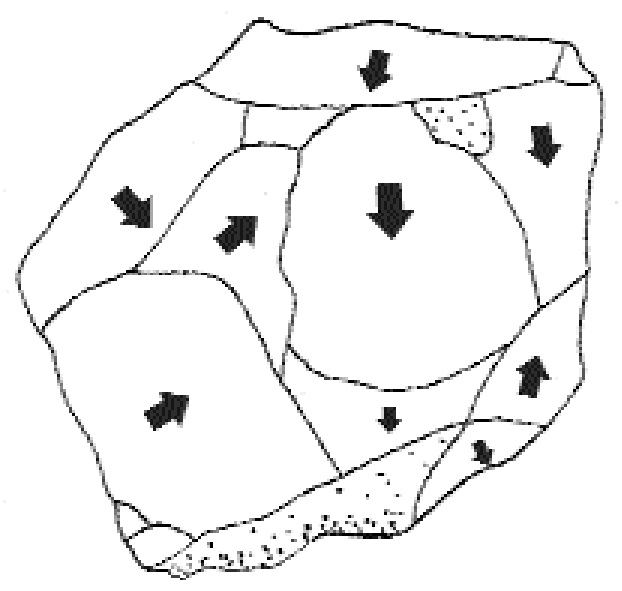

2

antérieures. Elle est particulièrement bien adaptée au débitage de nucléus sur éclat, de débris et de petits rognons irréguliers.

Un deuxième groupe important (12 pièces) est celui des nucléus globulaires (Bordes 1961) ayant subi une chaîne opératoire plus ou moins longue, ce qui permet de reconnaître les gestes utilisés, consistant en la succession de séries d'enlèvements unipolaires ou centripètes sur plusieurs surfaces (fig. 15). Le nucléus est tourné de nombreuses fois à la recherche d'un angle favorable dès que la surface de débitage exploitée n'offre plus de possibilité
Figure 13 - La Micoque, layer 6: 1 Levallois core, 2 discoid core. satisfaisante de production d'éclat. La plupart du temps, une seule surface de débitage, faite d'un seul enlèvement, est mise en place avant le commencement de l'exploitation, les autres séries d'enlèvements utilisant les angles préexistants. II arrive cependant qu'une surface naturelle soit utilisée comme plan de frappe initial ou que plusieurs plans de frappes soient mis en place. Ce n'est qu'après avoir épuisé toutes les possibilités que le nucléus est rejeté, que ce soit en raison de sa taille, de l'absence d'angles appropriés ou de la mauvaise qualité de la matière première. L'aspect des nucléus appartenant à ce groupe est 

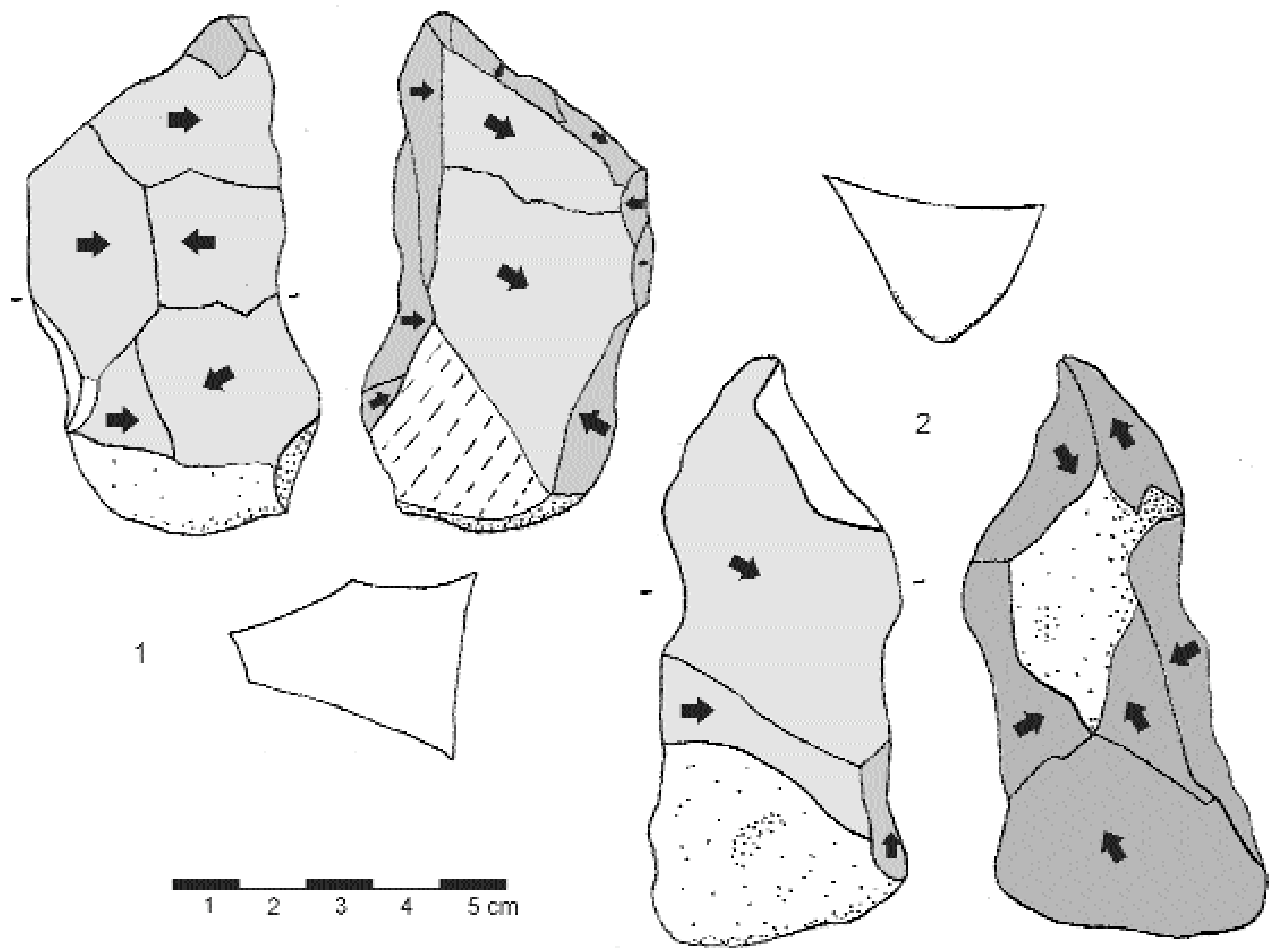

Figure 14 - La Micoque, couche 6 : nucléus en forme de lingot.

Figure 14 - La Micoque, layer 6 : ingot-shaped cores.

très variable et dépend de la forme initiale de la matière première et de l'intensité du débitage. Ce mode de débitage est particulièrement bien adapté au débitage d'éclats sur galets de petite et moyenne taille ainsi que sur fragments de matière première, mais des rognons de plus grande taille ont été également débités de cette façon. Les produits issus de cette production sont aussi bien des éclats ordinaires que de nombreux éclats à morphologie débordante, portant en fonction de l'avancement du débitage sur la face dorsale des négatifs unipolaires, bipolaires, croisés ou centripètes. II s'agit d'un système s'entretenant lui-même où chaque éclat est à la fois prédéterminé et prédéterminant.

Un grand nombre de nucléus informes (11 pièces) et de nucléus sur éclat (7 pièces) ne pouvant être classifiés dans aucun des types décrits ci-dessus est également présent. En général, la taille des nucléus ne peut être mise en relation avec le type de débitage. Toutes les grandes pièces ont en commun soit une mauvaise qualité de la matière première soit un accident de taille rendant le débitage impossible. Le rejet du nucléus dépend donc de l'impossibilité technique de produire des éclats acceptables après avoir épuisé toutes les possibilités. Aucun nucléus n'a été modifié pour servir d'outil après son rejet et aucune trace d'utilisation n'a pu être observée, bien qu'il faille remarquer que l'état de conservation des pièces est souvent mauvais.

\section{3 - Produits de débitage}

Les cassons n'ont jamais été modifiés. Ils ne seront donc pas considérés ici.

Une grande partie du débitage doit avoir eu lieu in situ. Le nombre de 12 produits pour un nucléus ${ }^{2}$ et la présence de nombreux éclats corticaux, dont $9 \%$ d'entames, indique que tous les stades du débitage doivent avoir eu lieu sur

(2) Toutes les valeurs mentionnées ont été calculées après avoir soustrait la fragmentation de l'industrie selon la méthode de Moncel (1999). 


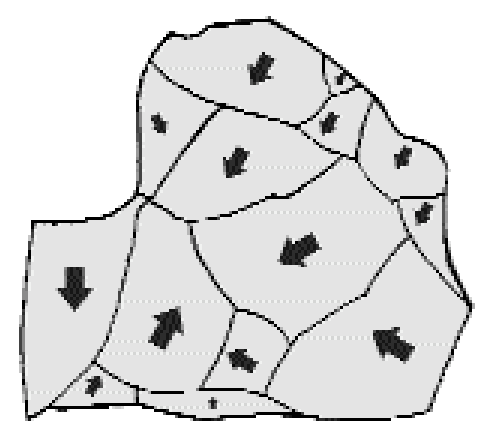

Figure 15 - La Micoque, couche 6 : nucléus globulaires.

Figure 15 - La Micoque, layer 6: globular cores.
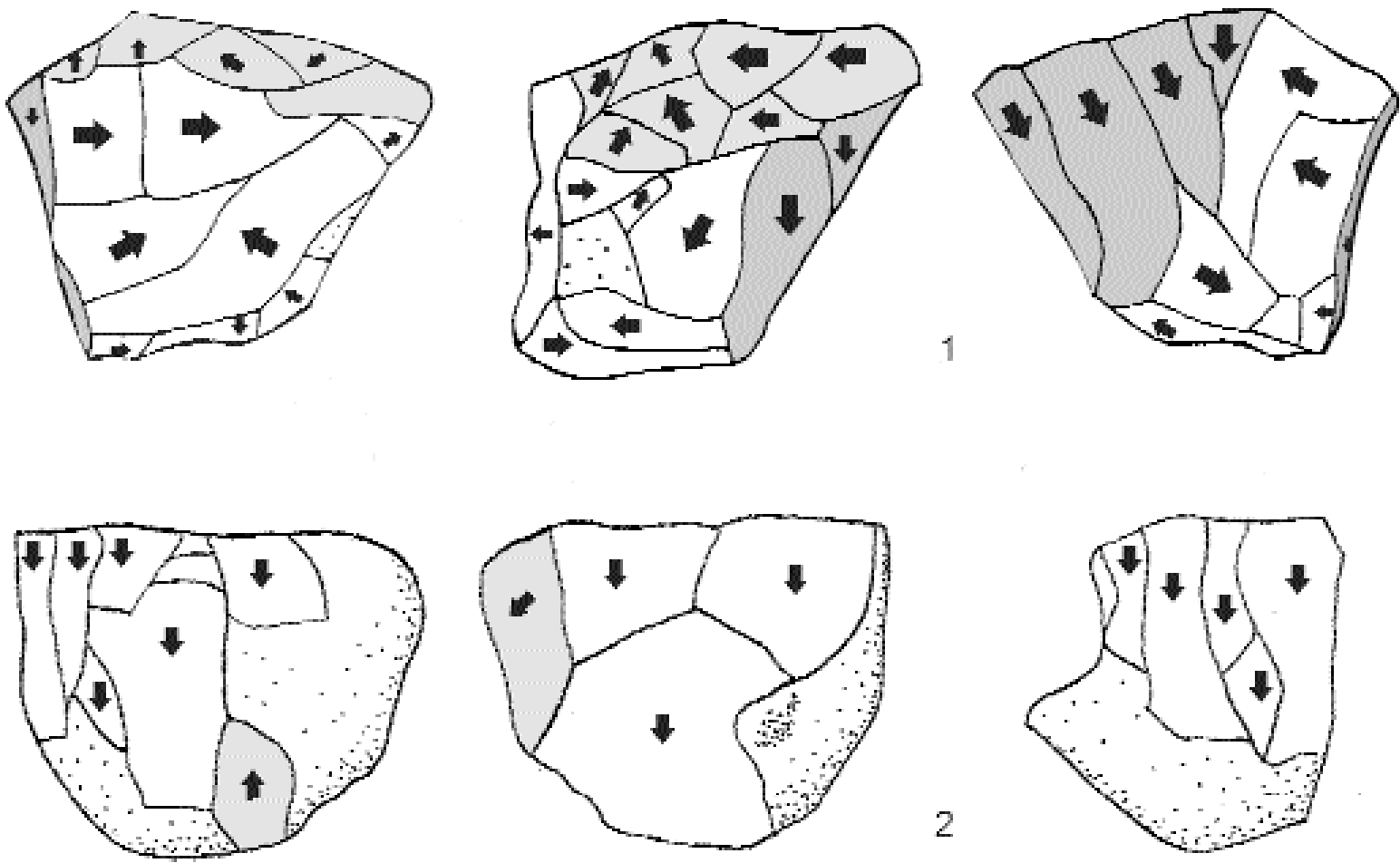

2

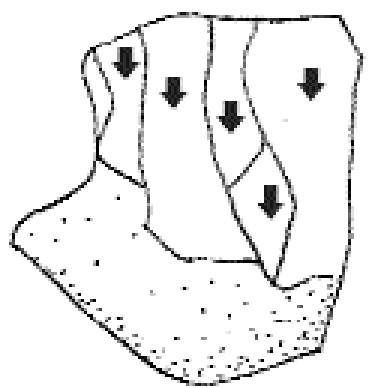

place, bien que les pièces de petites dimensions n'aient pas été collectées en raison de la méthode de fouille.

Près de $28 \%$ des produits de débitage sont des éclats à morphologie débordante, mais aucune de leurs caractéristiques métriques ou autres ne les différencie de façon significative des autres éclats. Ils peuvent être considérés comme des enlèvements résultant du débitage des nucléus globulaires et non comme des produits recherchés en raison de leur morphologie paticulière. Le débitage visait à la production d'éclats à peu près aussi longs que larges, dont la forme importait peu. Seuls deux éclats à morphologie Levallois ont pu être identifiés (fig. 16, 12 ), ce qui ne surprend pas vu la rareté des nucléus Levallois.

Les négatifs présents sur les faces dorsales des éclats confiment les observations faites sur les nucléus globulaires, à savoir que le débitage est au départ unipolaire pour devenir centripète au fil de la réduction. En effet, les éclats du débitage unipolaire sont les plus fréquents et ils sont significativement plus grands que les éclats du débi- tage centripète. Ils portent également moins de négatifs et sont plus souvent corticaux. Le débitage bipolaire est présent mais plus rare.

\section{4 - Outils sur éclat}

La proportion de pièces retouchées dans la collection Wiegers est de $12 \%$. Quinze pour cent des éclats et $8 \%$ des éclats à morphologie débordante sont retouchés, ce qui signifie que ces derniers n'ont pas été particulièrement recherchés pour les transformer en outils.

Les racloirs (fig. 17, 1-4, 6-7), très variés, sont avec $54 \%$ les pièces de loin les plus nombreuses. Outre les pièces de grandes dimensions et à retouche couvrante, bien représentées dans les collections d'Hauser, de nombreux racloirs simples sont présents ainsi que les pointes moustériennes. Sur les pièces de grandes dimensions, les encoches et amincissements supplémentaires sont fréquents.

Les encoches et denticulés forment $17 \%$ des outils sur éclats, et ce bien que toutes les pièces roulées et / ou concassées aient été écartées d'office. 

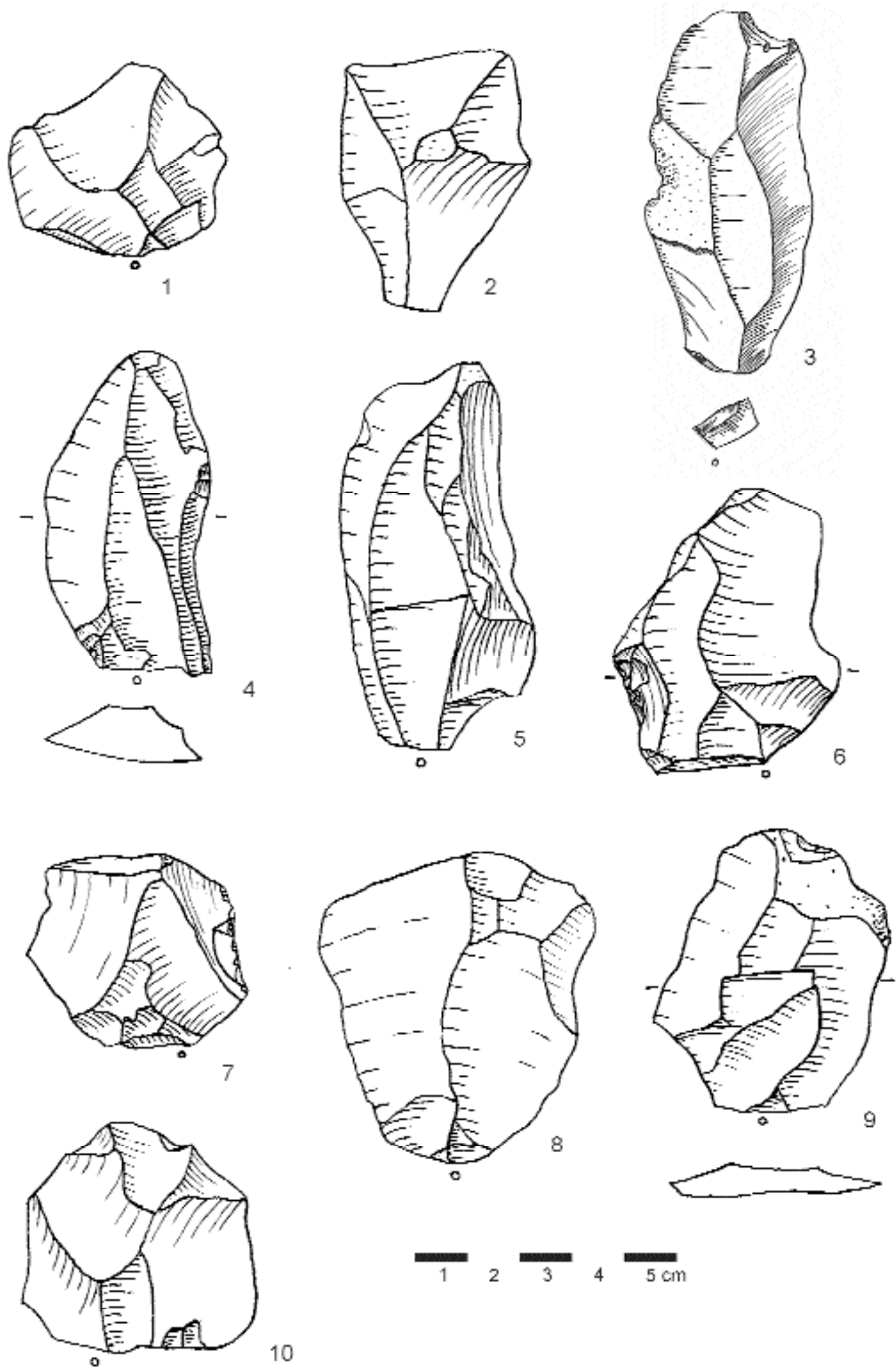

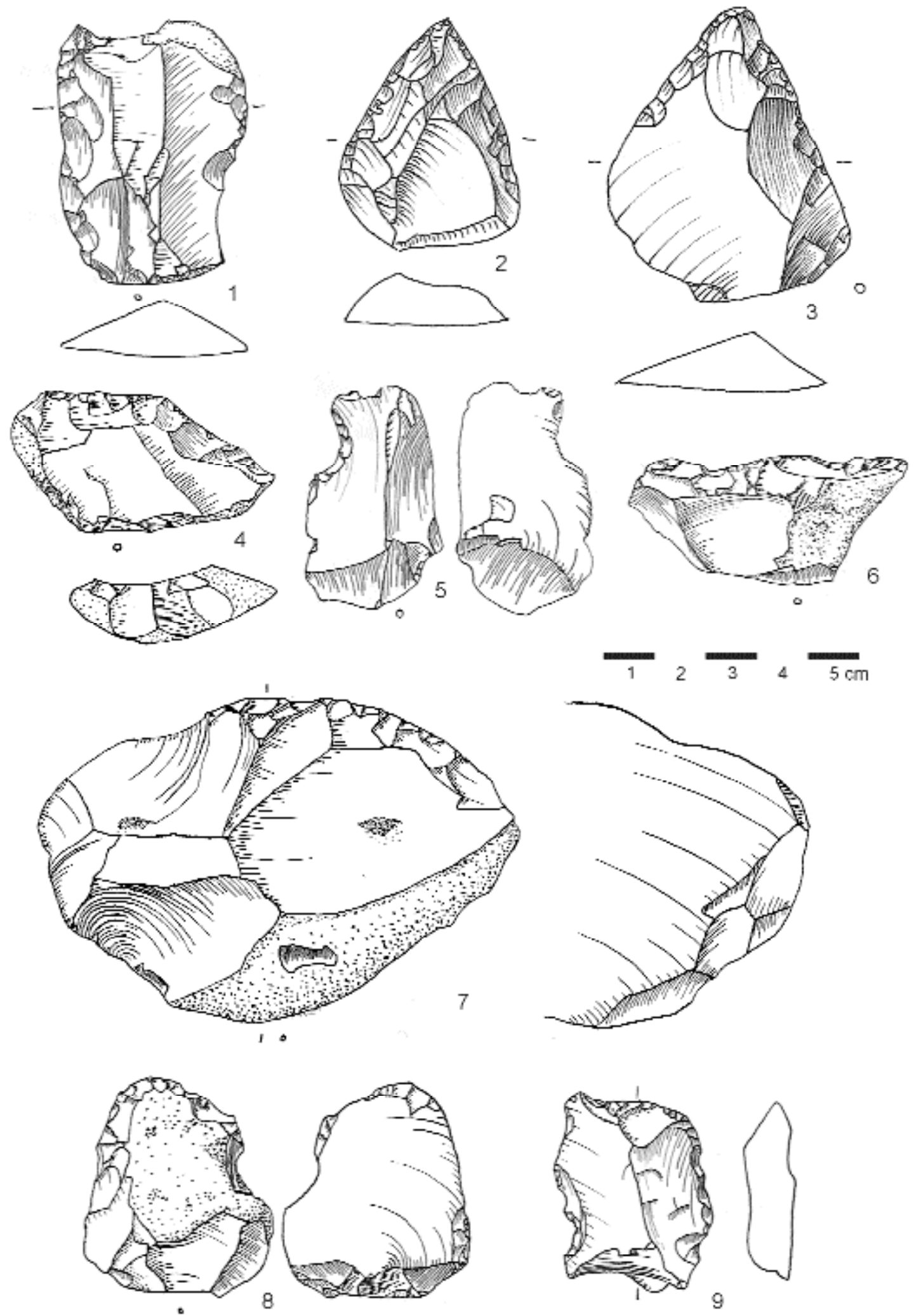

Figure 17 - La Micoque, couche 6 : 1-4 et 6-7 racloirs, 8-9 grattoirs, 5 perçoir.

Figure 17 - La Micoque, layer 6: 1-4 and 6-7 side-scrapers, 8-9 end-scrapers, 5 borer. 
Les grattoirs (8 \%) sont atypiques, jamais en bout de lame ou d'éclat allongé, mais utilisant le plus souvent une irrégularité dans la forme de l'éclat (fig. 17, 8-9).

Les perçoirs (4\%) portent des traces d'utilisation typiques. Leur pointe a été dégagée par encoches (fig. 17, 5).

II existe également toute une série de pièces atypiques retouchées sur la face ventrale ou à talon enlevé (15\%), dont il est difficile de dire si elles n'ont pas, du moins en partie, servi de nucléus (Bemard-Guelle et Porraz 2001).

Une seule pièce de petite taille peut être considérée comme outil multiple, si l'on exclut les denticulés et les racloirs multiples. II s'agit d'un petit perçoir à pointe cassée combiné à un grattoir sur talon. La pièce présente un dos à retouche abrupte dans le prolongement du grattoir.

\section{6 - L'INDUSTRIE DE LA COUCHE 7}

Elle ne contient aucune trace de la production ou de la présence d'outils bifaciaux.

\section{1 - Nucléus}

Les types présents sont identiques à ceux de la couche 6 . Le débitage Levallois (fig. 18, 1), rare, est représenté par un nucléus préférentiel et un nucléus récurrent centripète. Les nucléus discoïdes (fig. 18, 2-3), à une ou deux surfaces de débitage, sont au nombre de 8 , donc nettement plus fréquents que dans la couche 6 et tous épuisés. Le groupe des nucléus globulaires (fig. 19, 1-2) est comme dans la couche 6 le plus important et comporte 11 exemplaires de tous les types. Ils ont tous été rejetés en raison de leur petite taille ou de la mauvaise qualité de leur matière première. Seuls deux nucléus en forme de lingot sont présents (fig. 19, 3), quatre sont des nucléus informes et trois sur éclat.

La seule différence importante par rapport à la couche 6 est que les nucléus discoïdes sont plus nombreux que les nucléus en forme de lingot. Les types de débitage sont identiques et la taille des nucléus ne diffère pas significativement d'une couche à l'autre, ils sont tous exploités au maximum. Le type de matière première est identique dans les deux couches.

\section{2 - Produits de débitage}

Le nombre de 22 produits par nucléus et la présence d'une majorité d'éclats corticaux dont $14 \%$ d'entames indiquent ici aussi un débitage in situ. Les éclats sont à peu près aussi larges que longs. L'importance du débitage globulaire se traduit par un nombre important (25\%) d'éclats à morphologie débordante. Il est intéressant de noter que ceux-ci ont, en raison de leur morphologie, mieux résisté au concassage que les éclats ordinaires. Leur taille moyenne est, si l'on prend en compte l'ensemble des pièces, nettement plus élevée que celle des éclats, tandis qu'elle correspond à celle des produits de débitage de la couche 6 , soulignant le rôle du concassage dans l'aspect général de l'industrie de la couche 7, décrite par Wiegers et al. (1913) et Hauser (1916) comme «microlithique» et «primitive». Les caractéristiques des éclats à morphologies débordantes ne diffèrent cependant aucunement de celles des éclats ordinaires, ce qui indique qu'ils représentent dans cette couche également des produits normaux du débitage globulaire.
Un seul produit de morphologie Levallois, une lame Levallois (fig. 16, 3), a pu être identifié.

\section{3 - Outils sur éclat}

Seule la collection Wiegers contient des pièces retouchées en provenance de la couche 7 , probablement parce qu'elle ne contenait aucune belle pièce de grande taille.

$16 \%$ des supports ont été retouchés, à savoir $18 \%$ des éclats ordinaires et $11 \%$ des éclats à morphologie débordante.

Les racloirs (fig. 20, 1-6) sont ici aussi les pièces les plus nombreuses (46) et représentent $49 \%$ des outils sur éclat. II s'agit principalement de racloirs latéraux mais les déjetés sont également nombreux tandis que les racloirs transversaux sont plus rares. Les racloirs abrupts sont également présents.

Les grattoirs, au nombre de 13, sont relativement fréquents. Les pièces typiques ont été réalisées sur éclats allongés ou éclats laminaires et présentent parfois une cassure de l'extrémité proximale. Les grattoirs typiques (fig. 20,7-8) sont presque aussi fréquents que les grattoirs atypiques.

Les encoches et denticulés, très hétérogènes, sont au nombre de neuf. En raison du fort concassage des objets de la couche 7 , seules les pièces intactes ont été prises en considération. II se pourrait donc que ce groupe soit sousreprésenté.

Un seul perçoir, atypique, est présent. II est intéressant de noter la présence d'un groszak, considéré comme typique du Micoquien (Richter 1997). Les pièces portant une retouche atypique, touchant soit la face ventrale soit le talon, sont au nombre de vingt. II existe quatre pièces ayant un dos retouché opposé à un tranchant laissé brut (fig. 20, 9-10). II est possible que ce tranchant porte des traces d'utilisation, mais les conditions d'enfouissement des artéfacts interdisent toute conclusion.

\section{7- L'INDUSTRIE DE LA COUCHE 8}

Une seule pièce bifaciale atypique et de petite taille provient de cette couche (fig. 21, p.184).

\section{1 - Nucléus}

Les nucléus Levallois (fig. 22), au nombre de cinq, sont plus nombreux et plus typiques que dans les autres couches. Leur taille est inférieure à celle des nucléus Levallois des couches 6 et 7, semblant indiquer une meilleure maîtrise de la technique permettant une utilisation plus intense des rognons. Plusieurs types sont présents, préférentiel ou récurrent, à débitage unipolaire, bipolaire ou centripète.

Les nucléus discoïdes (fig. 23), au nombre de sept, sont également variés. Onze nucléus sont de type globulaire (fig. 24,1 ) et couvrent toutes les variantes de ce groupe. Les nucléus en forme de lingot (fig. 24, 2) sont de nouveau nombreux (10), tandis que seuls trois sont informes. Sept pièces sont des nucléus sur éclat.

Mise à part une augmentation significative du point de vue statistique des nucléus discoïdes par rapport à la couche 6 , il n'existe pas de différence entre les proportions de 

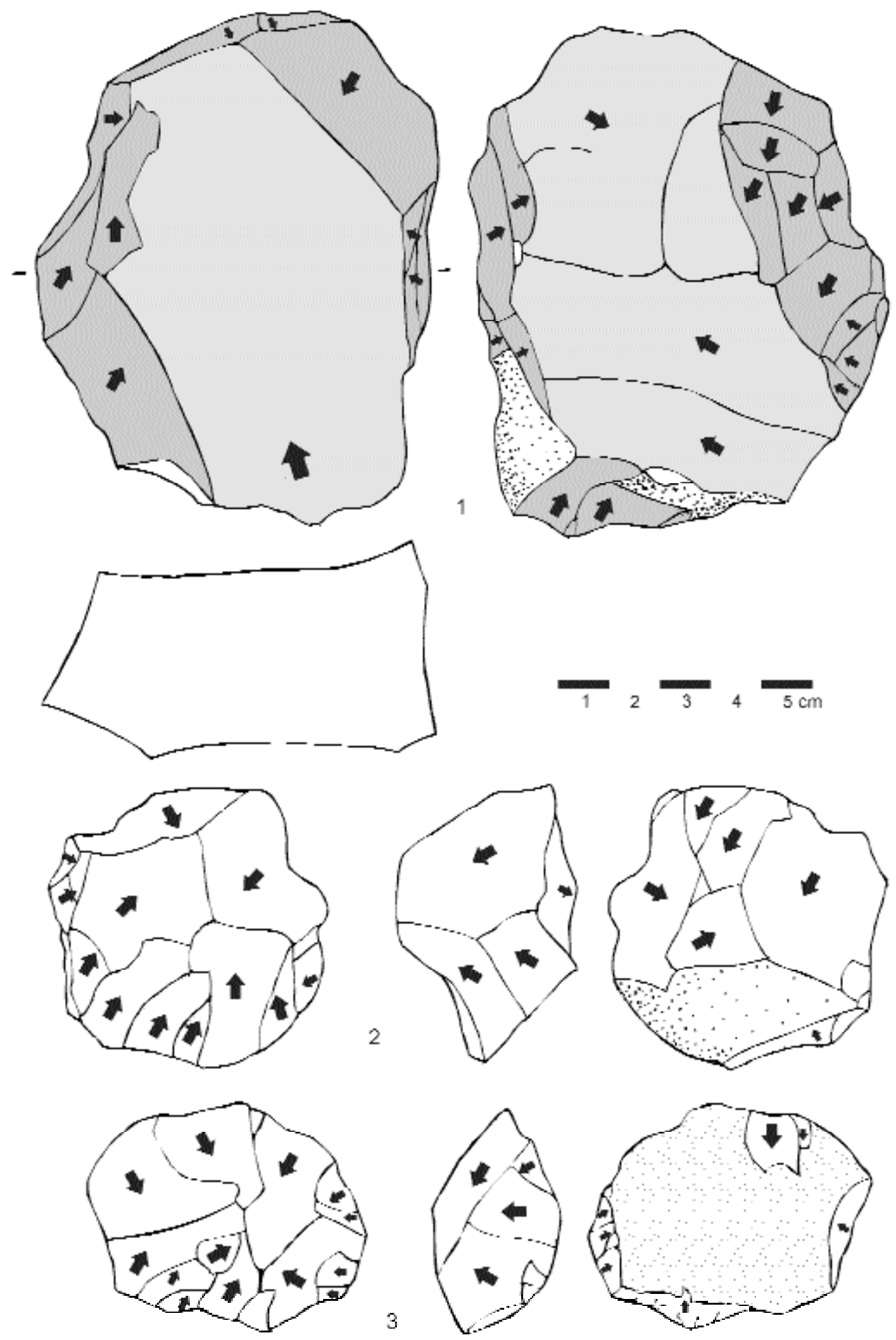

Figure 18 - La Micoque, couche 7 : 1 nucléus Levallois, 2-3 nucléus discoïdes.

Figure 18 - La Micoque, layer 7: 1 Levallois core, 2-3 discoid cores. 

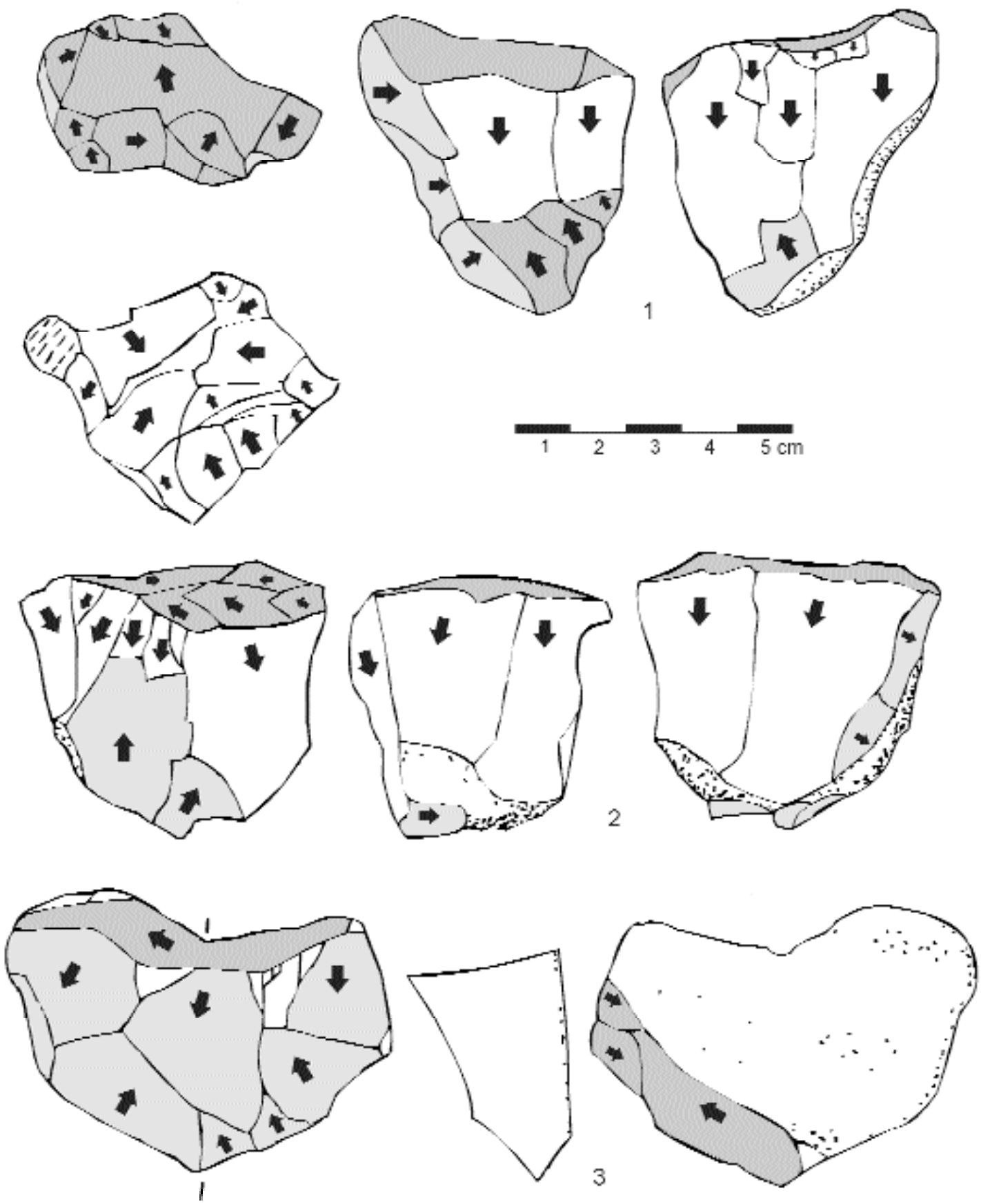

Figure 19 - La Micoque, couche 7 : 1-2 nucléus globulaires, 3 nucléus en forme de lingot.

Figure 19 - La Micoque, layer 7: 1-2 globular cores, 3 ingot-shaped core. 

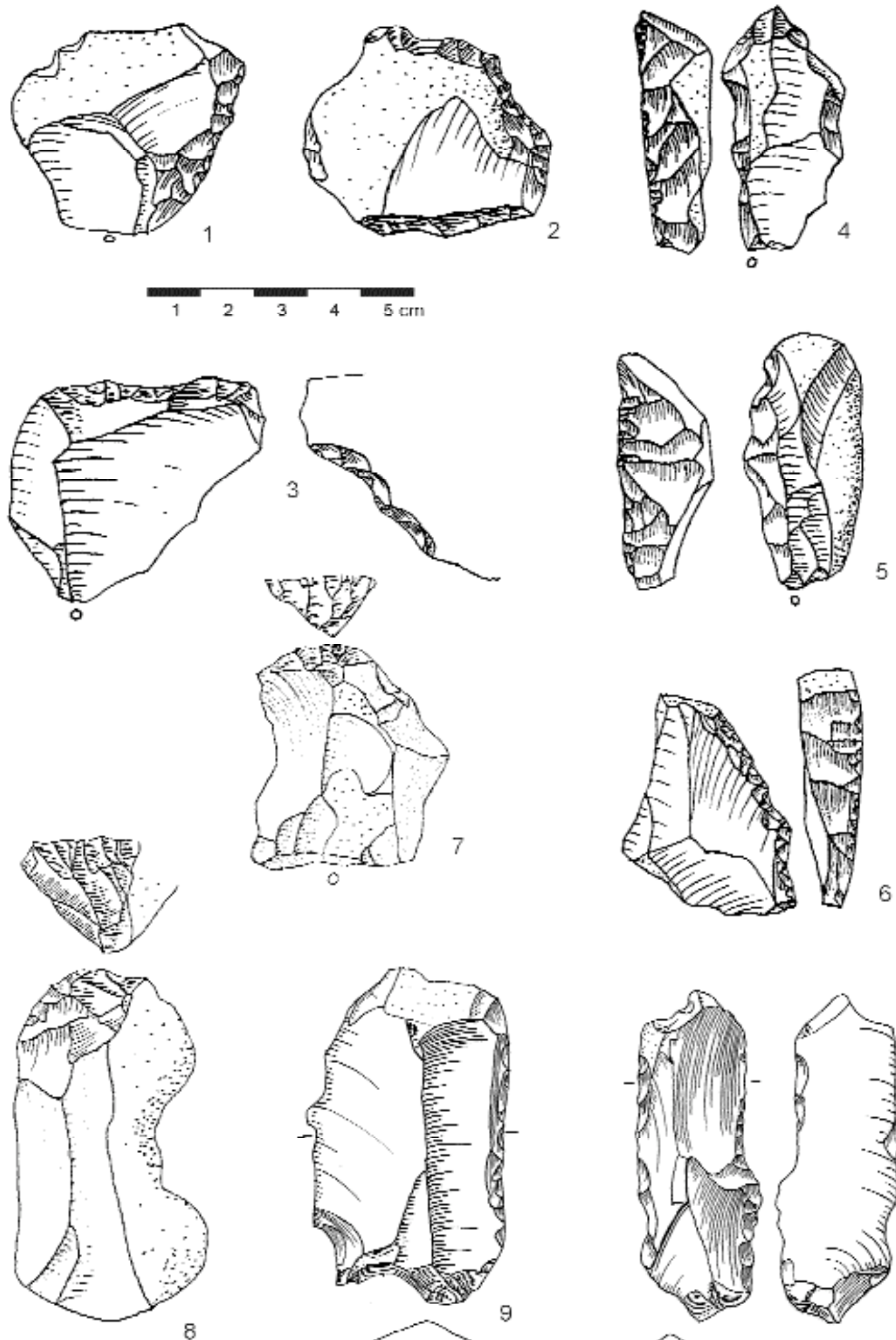

8
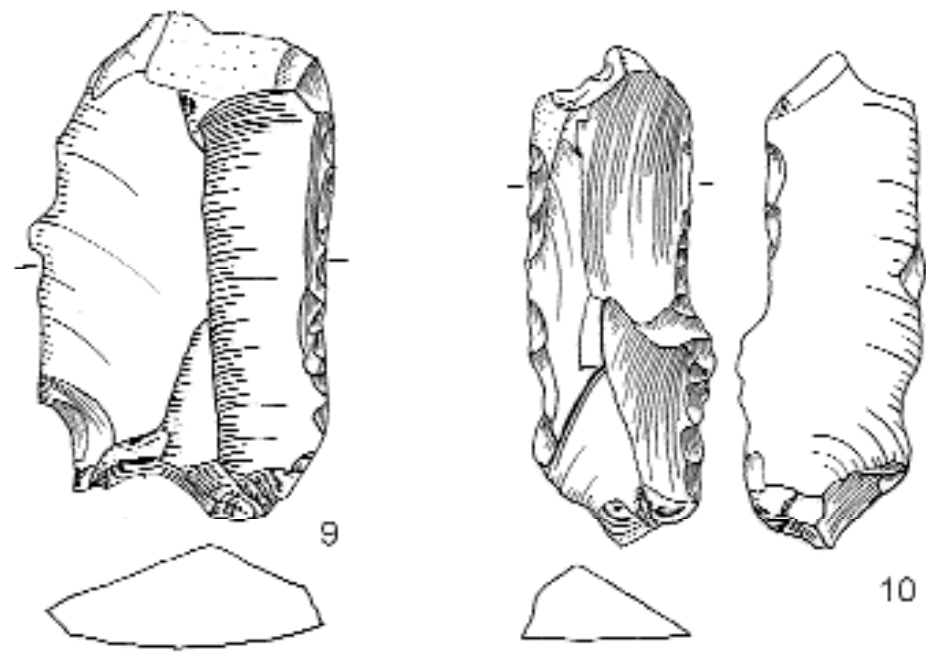

Figure 20 - La Micoque, couche 7 : 1-6 racloirs, 7-8 grattoirs, 9-10 “couteaux ”.

Figure 20 - La Micoque, layer 7: 1-6 side-scrapers, 7-8 end-scrapers, 9-10 “knifes ”. 


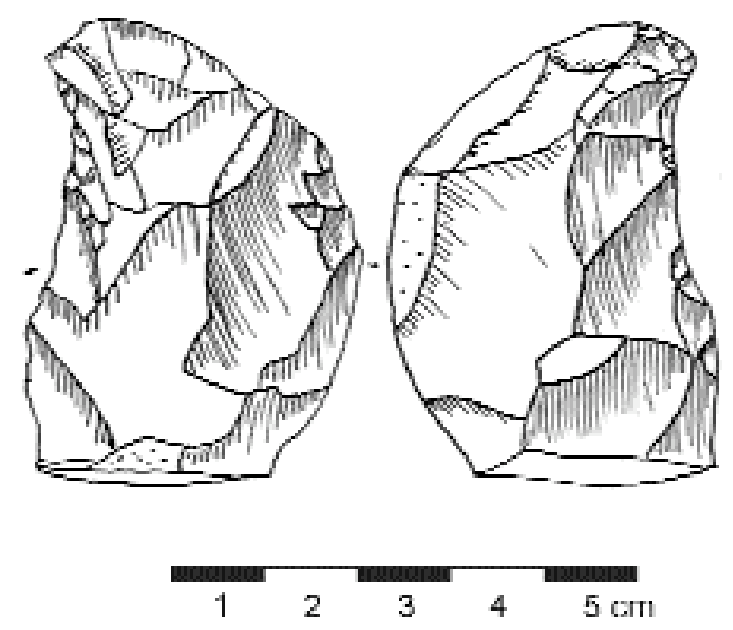

types de nucléus dans les couches. L'augmentation du nombre de nucléus Levallois n'est, quant à elle, pas significative d'un point de vue statistique. La taille des négatifs indique cependant que les enlèvements prédéterminés sont nettement plus grands que tous les autres éclats.

\section{2 - Produits de débitage}

Ici aussi, le débitage doit avoir eu lieu in situ car la proportion de produits par rapport au nombre de nucléus est de 17:1. Les éclats corticaux ainsi que les entames sont un peu moins nombreux que dans les autres couches mais pas de façon significative. La proportion d'éclats à morphologie débordante $(25 \%)$ correspond exactement à celle des couches 6 et 7 mais ces pièces sont nettement plus grandes que les éclats ordinaires. On assiste à un allongement significatif des produits de débitage (1,2 au lieu de 1) et les pièces Levallois sont au nombre de 7 (fig. 16, 4-10). Les directions de débitage reconnaissables sur les éclats correspondent aux observations faites pour les deux autres couches.

\section{3 - Outils sur éclat}

Une seule pièce provenant de cette couche a pu être trouvée en dehors de la collection Wiegers. II s'agit d'un très grand racloir sur éclat Levallois conservé dans les collections des Reiss-Engelhorn-Museen de Mannheim (fig. 25, 1).

Des produits de débitage (16,5\%) ont été retouchés dont $25 \%$ d'éclats à morphologie débordante.

Les outils les plus fréquents sont les racloirs (59), dominés par les racloirs latéraux simples (fig. 25, 2-6). Seules certaines grandes pièces portent une retouche envahissante sur la face ventrale.

Les encoches et denticulés, au nombre de 14, forment un groupe très inhomogène.

Les 13 grattoirs sont presque tous typiques. Ils sont faits sur petits éclats ronds ou quadrangulaires ou sur éclats laminaires (fig. 26, 1-3).

II n'existe aucun perçoir dans cette couche mais les burins, au nombre de trois, font leur apparition. Ils sont cependant atypiques (fig. 26, 4-5).

Près du quart des pièces retouchées (29) sont des éclats portant une retouche sur la face ventrale ou un talon enlevé n'appartenant à aucun type connu.
Figure 21 - La Micoque, couche 8 : outil bifacial.

Figure 21- La Micoque, layer 8: bifacial tool.

\section{8 - DISCUSSION}

Ce bref aperçu des ensembles lithiques provenant des couches 6,7 et 8 de La Micoque permet quelques comparaisons. Les méthodes de débitage présentes sont identiques dans les trois couches, où les nucléus globulaires dominent toujours, mais il apparaît que l'importance des autres méthodes de débitage varie. II est cependant délicat de tirer des conclusions définitives quant à la signification de cette variabilité étant donné les problèmes inhérents à la collecte des pièces : il ne s'agit pas de la totalité de l'industrie mais bien d'un échantillon. II semblerait pourtant qu'une certaine augmentation des méthodes de débitage plus contrôlées, comme Levallois et Discoïde, puisse être observée entre les trois couches. En outre, la proportion d'éclats allongés et l'effectivité de l'exploitation des nucléus Levallois augmentent dans la couche 8.

La couche 6 est la seule à avoir livré des outils bifaciaux en grand nombre mais la question de leur production sur place ne peut être résolue avec le matériel conservé. Cet ensemble peut être mis en relation avec les Keilmessergruppen d'Europe centrale en raison de la présence de couteaux bifaciaux principalement et des grandes ressemblances avec les outils bifaciaux de Bockstein (Bosinski 1967 ; Wetzel et Bosinski 1969) qui n'est malheureusement pas daté (pour des avis divergents quant à la situation chronologique du site voir : Filzer 1969 ; Jöris 2002 ; Richter 1997 ; Schmid 1969).

D'un point de vue typologique, les couches 7 et 8 doivent être attribuées au Moustérien de type Quina bien que le grand nombre de grattoirs remplace une part des racloirs et donne un diagramme cumulatif quelque peu particulier. II faut cependant remarquer que le nombre d'outils sur éclat est inférieur à 100 (respectivement 69 pour la couche 6, 94 pour la couche 7 mais 128 pour la couche 8) et ne représente donc pas un groupe statistiquement significatif.

D'un autre côté, une très grande ressemblance subsiste entre les ensembles lithiques provenant de ces trois couches tant du point de vue technologique que typologique si l'on exclut les outils bifaciaux. 

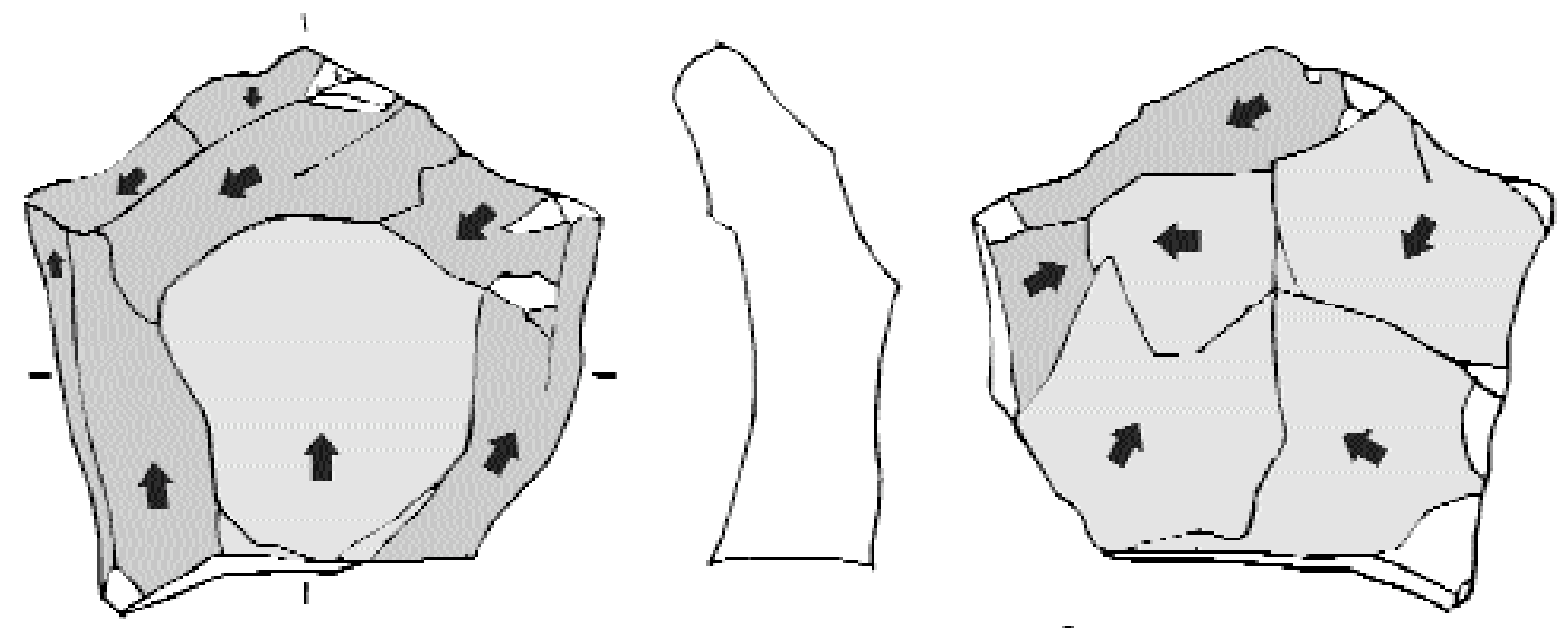

1
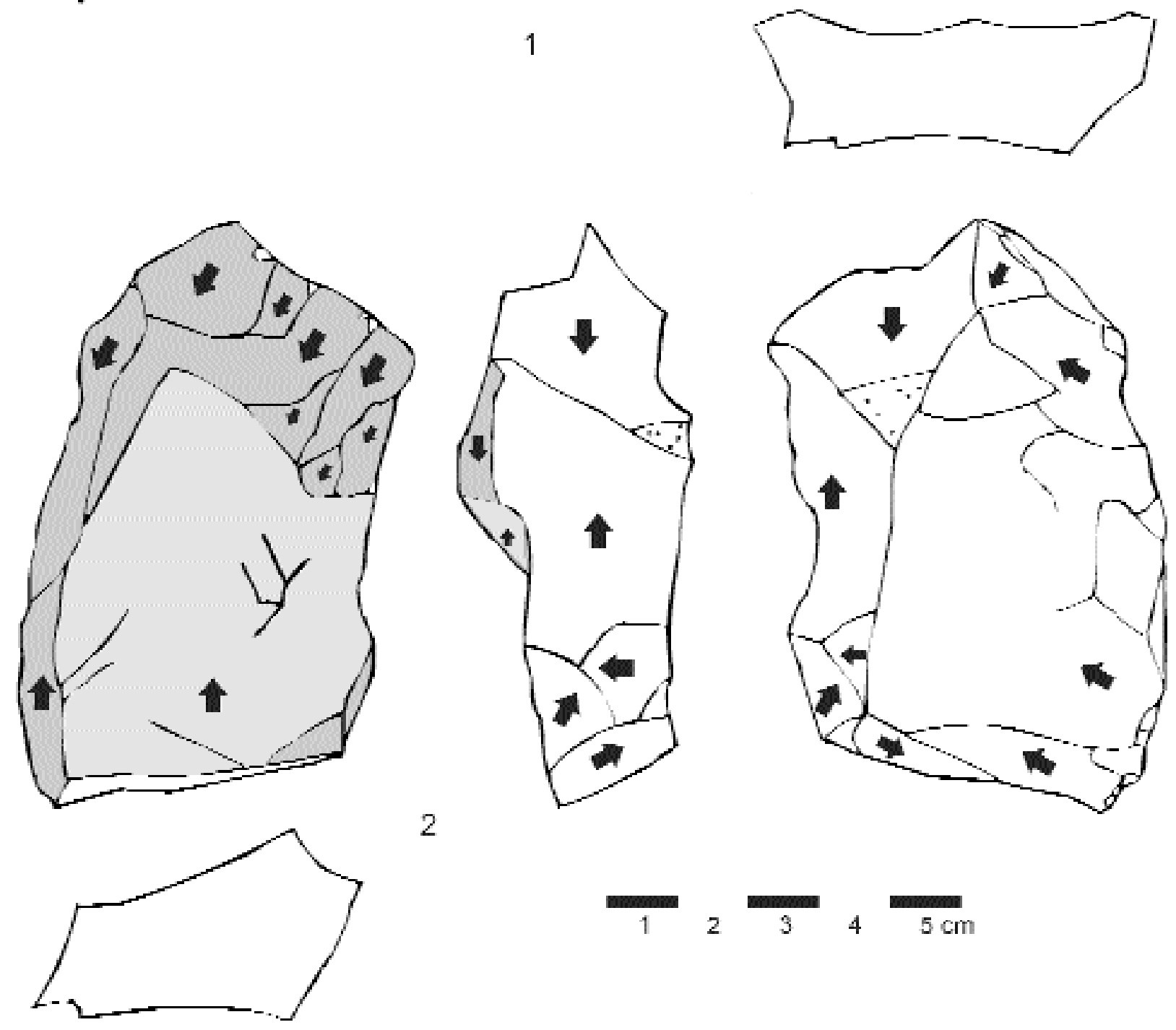

Figure 22 - La Micoque, couche 8 : nucléus Levallois.

Figure 22 - La Micoque, layer 8: Levallois cores. 

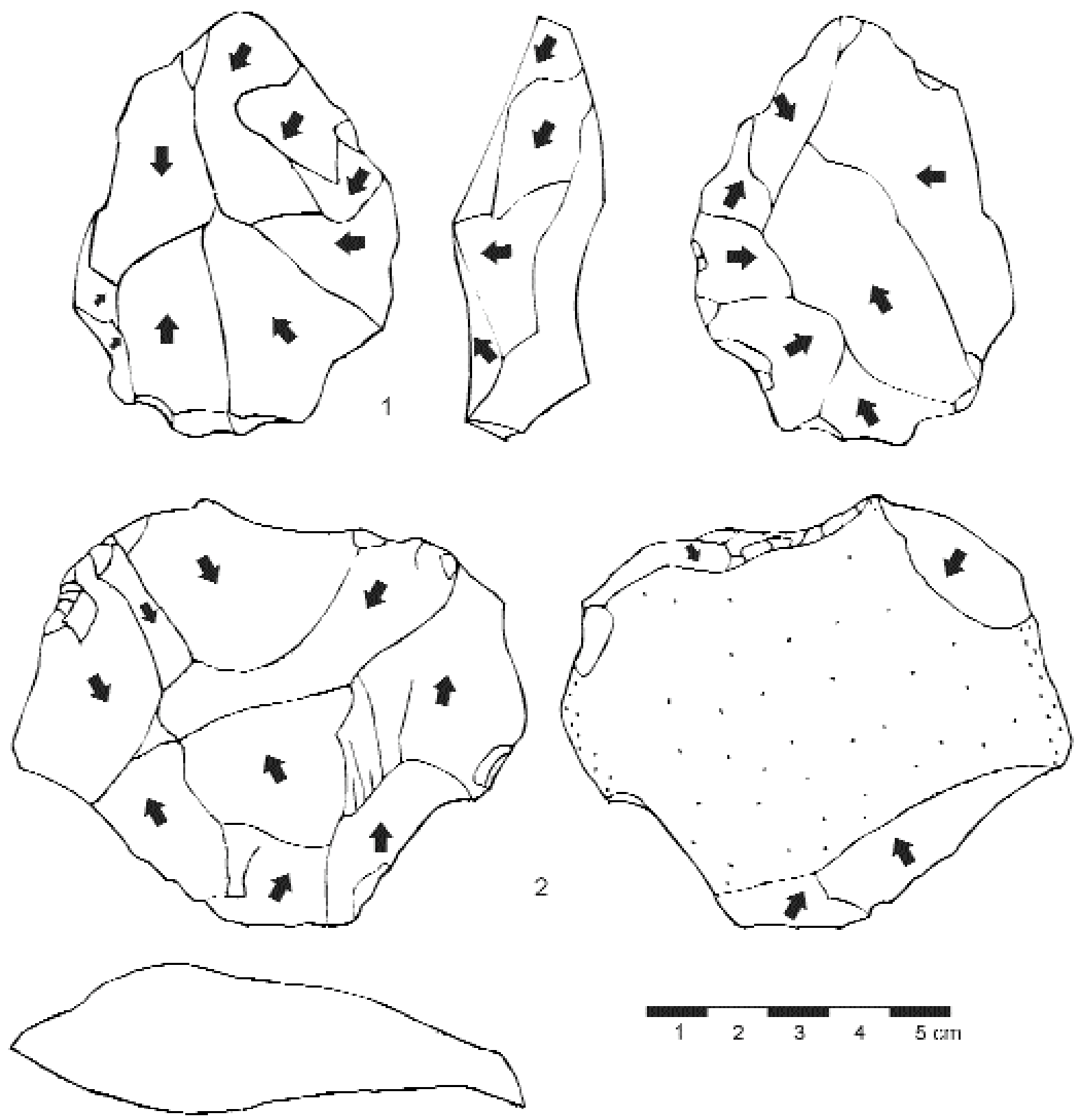

Figure 23 - La Micoque, couche 8 : nucléus discoïdes.

Figure 23 - La Micoque, layer 8: discoid cores.

\section{9- CONCLUSION}

La couche «micoquienne» de La Micoque (6) peut être intégrée dans le cycle de sédimentation décrit par Texier et Bertran (1993), étant surmontée d'une couche d'origine fluviatile (7), elle-même recouverte par un dépôt de pente (8) pouvant correspondre au DP4 observé sur le site. Son âge ne peut donc être fortement inférieur à celui des couches sous-jacentes. L'étude du contenu des couches 6,7 et 8 a mis en évidence une industrie de type

Paléolithique moyen à débitage varié mais principalement de type globulaire. La seule différence véritable consiste en la présence dans la couche 6 de nombreux bifaces absents dans les autres couches.

Ceci met en évidence la problématique inhérente à la signification donnée aux différents éléments définissant un inventaire telle la présence ou l'absence d'outils bifaciaux ou d'un type de débitage particulier pour la classification d'un ensemble lithique au Paléolithique moyen. 

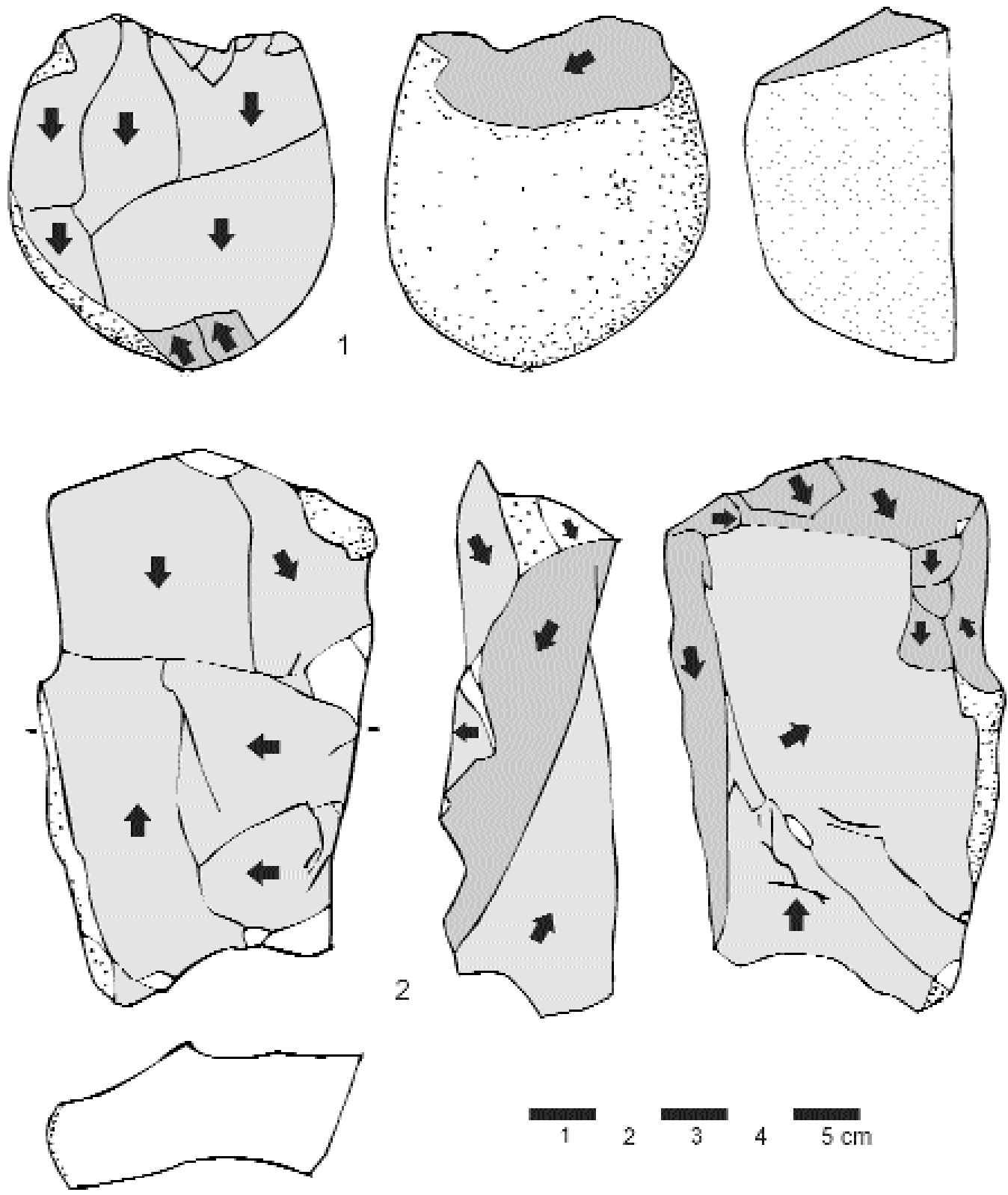

Figure 24 - La Micoque, couche 8 : 1 nucléus globulaire, 2 nucléus en forme de lingot.

Figure 24 - La Micoque, layer 8: 1 globular core, 2 ingot-shaped core.

Les facteurs conduisant à la production et l'utilisation d'outils bifaciaux par exemple peuvent être tellement variés que seule une étude détaillée de tous les aspects de l'occupation, telles la taille du groupe, la durée de séjour, les activités entreprises sur place, la qualité et l'accessibilité des matières premières, etc. pourrait permettre de distinguer le rôle joué par les concepts présents de celui joué par des circonstances périphériques. Une telle étude n'est pas faisable sur le matériel de La Micoque en raison des conditions de récolte.
Soit les concepts accessibles aux personnes ayant produit les différents ensembles lithiques étaient identiques mais n'ont été tous réalisés que dans la couche 6 , soit le concept des outils bifaciaux faisait défaut aux occupants des couches 7 et 8 qui utilisèrent cependant exactement les mêmes méthodes de débitage que leur prédécesseurs dans des proportions semblables, ainsi que les mêmes outils sur éclats. 

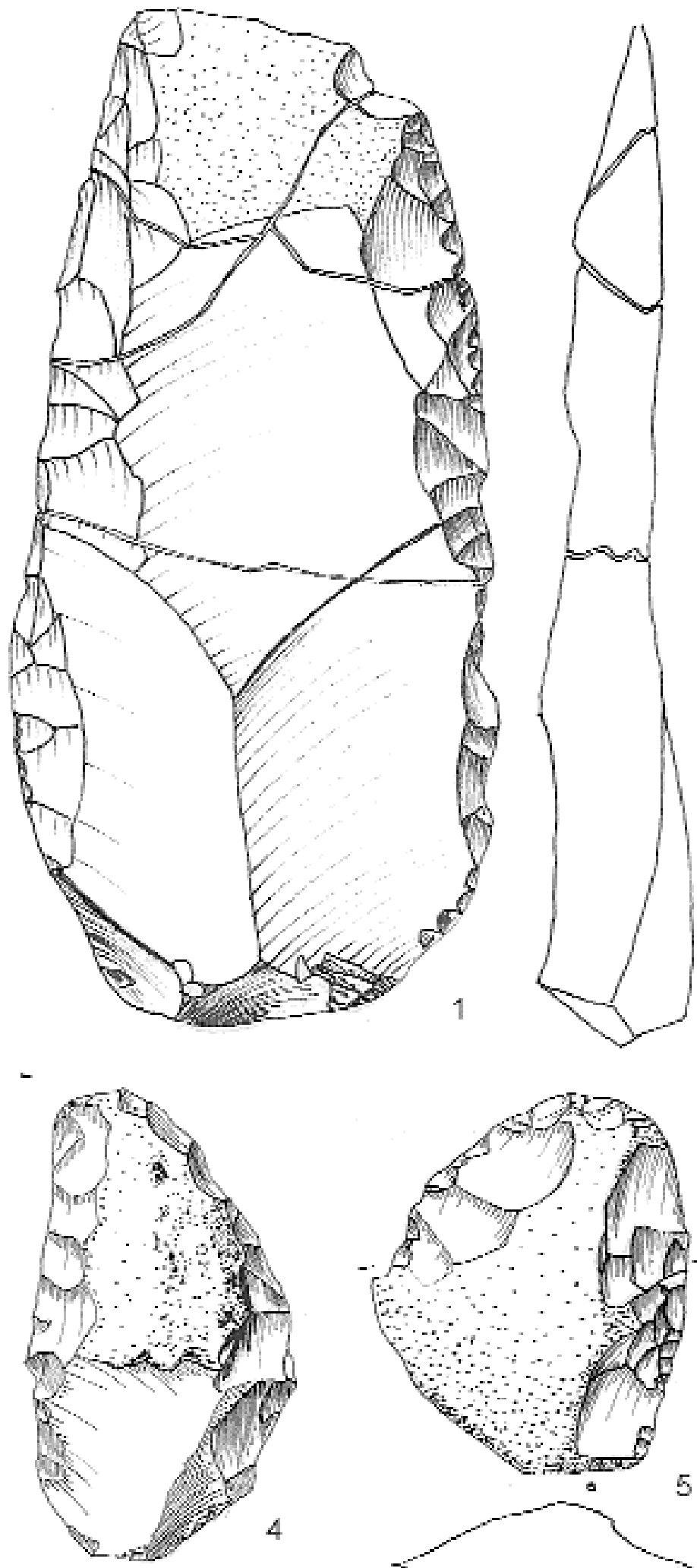
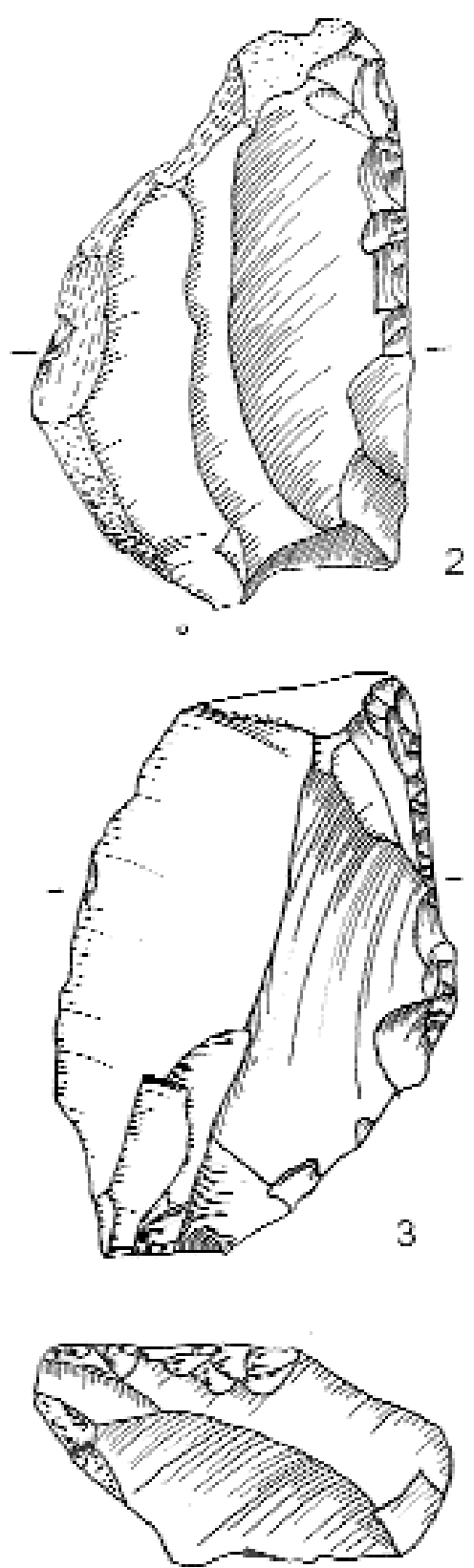

6
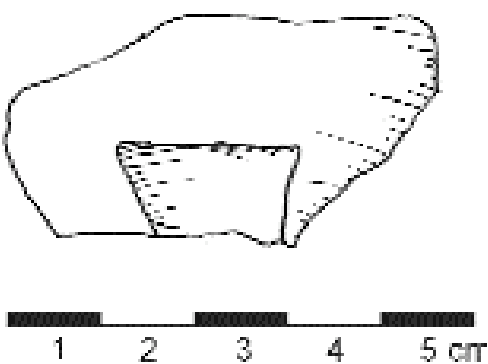

Figure 25 - La Micoque, couche 8 : 1 racloir sur éclat Levallois, 2-6 racloirs.

Figure 25 - La Micoque, layer 8: 1 side-scraper on Levallois flake, 2-6 side-scrapers. 

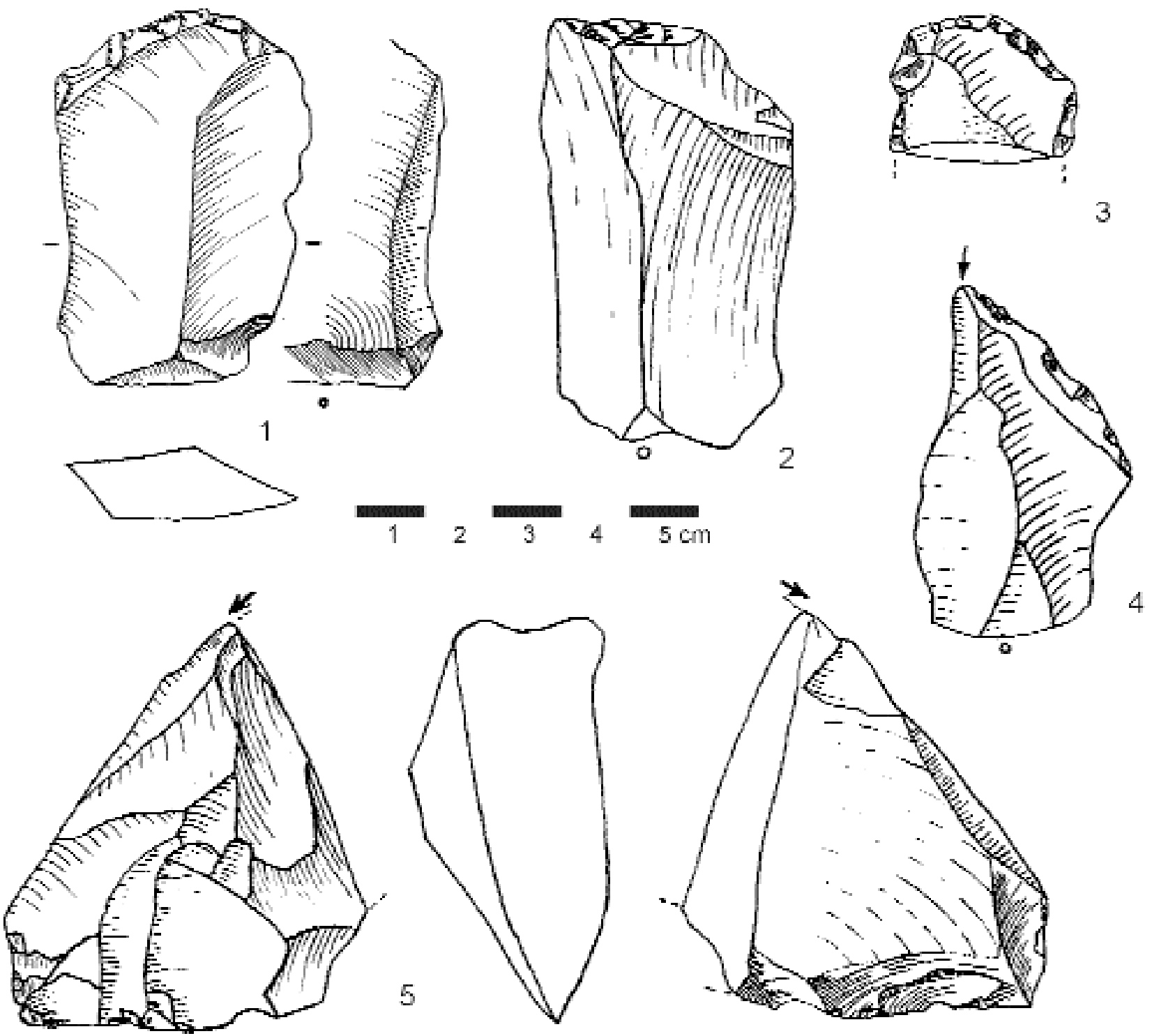

2

Figure 26 - La Micoque, couche 8 : 1-3 grattoirs, 4-5 burins.

Figure 26- La Micoque, layer 8: 1-3 end-scrapers, 4-5 burins.

\section{Remerciements}

Dr. K.-W. Beinhauer, Mannheim ; Dr. G. Böhme, Museum für Naturkunde, Berlin ; PD Dr. M. Bolus, Universität Tübingen ; D. Bonjean, Grotte Scladina ; Prof. Dr. G. Bosinski, Universität zu Köln ; Dr. K.H. Brandt, Bremen ; Prof. Dr. M. Brunet, Université de Poitiers ; Dr. J. M. Burdukiewicz, Université de Wroclaw ; Dr. C. Buret, Musée Ste Croix, Poitiers ; Dr. J. Burger, Universität Mainz ; Dr. J.-J. Cleyet-
Merle, Musée National de Préhistoire, Les Eyziesde-Tayac ; Curt-Engelhorn Stiftung, Mannheim ; R. Drößler, Zeitz ; Prof. Dr. L. Fiedler, Marburg ; Dr. G. Garcia, Université de Poitiers ; Dr. J.-M. Gouédo, Paris ; Dr. J. Grünberg, Landesamt für Archäologie Sachsen-Anhalt mit Landesmuseum für Vorgeschichte, Halle ; A. Heinke, Dipl.-Geol., Bundesanstalt für Geowissenschaften und Rohstoffe, Dienstbereich Berlin ; B. Henriette, Les Eyzies-de-Tayac ; A. Hoffmann, M.A., Museum für 
Vor- und Frühgeschichte, Schloß Charlottenburg, Berlin ; Prof. Dr. K.-D. Jäger, Halle ; Dr. A. Justus, Monrepos ; B. Kaulich †, M.A., Nürnberg ; Prof. Dr. W. Menghin, Museum für Vor- und Frühgeschichte, Berlin Charlottenburg ; Dr. A. Morala, Musée National de Préhistoire, Les Eyzies-de-Tayac ; Dr. G. Morgenroth, Universität Erlangen ; Dr. B. Mühldorfer, Vorgeschichtliche Sammlung der Naturhistorischen Gesellschaft, Nürnberg ; H. Orth, Ratingen ; Prof. Dr. M. Otte, Université de Liège ; Dr. A. Pastoors, Neanderthal Museum, Mettmann ; Prof. Dr. A. Pletsch, Universität Marburg ; Prof. Dr. L. Reisch, Universität Erlangen ; Dr. U. Reuter, Landesamt für Archäologie mit Landesmuseum für Vorgeschichte, Dresden ; Prof. Dr. J. Richter, Universität zu Köln ; Dr. W. Rosendahl, ReissEngelhorn-Museen Mannheim ; Dr. C. Schwab, Musée des Antiquités Nationales, St. Germain-enLaye ; Prof. Dr. W. Schirmer, Düsseldorf ; Dr. G. Tromnau, Duisburg ; Dr. Habil. A. Turq, Musée National de Préhistoire, Les Eyzies-de-Tayac ; Dr. M. Ulrix-Closset, Lüttich ; Dr. Th. Uthmayer, Universität zu Köln ; Dr. St. Veil, Niedersächsisches Landesmuseum Hannover ; R. Walter M.A., Schelklingen ; PD Dr. T. Weber, Magdeburg ; D. Wegner, Neckarbischofsheim ; PD Dr. W. Weißmüller $\dagger$, Universität Erlangen ; K. Werberger M.A., Museum UIm ; Prof. Dr. A. Wieczorek, ReissEngelhorn-Museen, Mannheim ; Dr. C. Züchner, Universität Erlangen.

Un merci tout particulier aux rapporteurs, Messieurs P rof. Dr. J.-P. Texier et Dr. P.-J. Texier, pour leurs critiques constructives.

\section{BIBLIOGRAPHIE}

BERNARD-GUELLE S., PORRAZ G. 2001 - Amincissement et débitage sur éclat: définitions, interprétations et discussion à partir d'industries lithiques du Paléolithique Moyen des Préalpes du Nord françaises. Paléo, 13, p. 53-72.

BIRKNER F. 1918 - Die Kultur von La Micoque. Wiener Prähistorische Zeitschrift, 5, p. 1-13.

BOËDA E. 1993 - Le débitage discoïde et le débitage Levallois récurrent centripète. Bulletin de la Société Préhistorique Française, 90 (6), p. 392-404.

BOËDA E. 1995 - Caractéristiques techniques des chaînes opératoires lithiques des niveaux micoquiens de K_Ina (Tchécoslovaquie). In : Les industries à pointes foliacées d'Europe Centrale, Actes du Colloque de Miskolc, Les Eyzies-de-Tayac, p. 57-72 (Paléo supplément 1).

BOËDA E. 2001 - Détermination des unités techno-fonctionnelles de pièces bifaciales provenant de la couche acheuléenne C'3 base du site de Barbas I. In : Cliquet D. (Dir.), Les industries à outils bifaciaux du Paléolithique moyen d'Europe occidentale. Actes de la table-ronde internationa- le organisée à Caen (Basse-Normandie, France) - 14 et 15 octobre 1999, Liège, p. 51-75 (ERAUL 98).

BORDES F. 1961 - Typologie du Paléolithique ancien et moyen. Paris : Presses du CNRS, 224 p.

BORDES F. 1984a - Notions de géologie quatemaire. Leçons sur le Paléolithique Tome 1, Cahiers du Quatemaire 7, Paris : CNRS, Centre régional de publication de Bordeaux, 288p.

BORDES F. 1984b - Le Paléolithique en Europe. Leçons sur le Paléolithique Tome 2, Cahiers du Quatem a ire 7, Paris : CNRS, Centre régional de publication de Bordeaux, 459p.

BOSINSKI G. 1967 - Die mittelpaläolithischen Funde im westlichen Mitteleuropa. Institut für Ur- und Frühgeschichte der Universität zu Köln , Köln-Graz : Böhlau Verlag, 203 p., 197 pl, 7 cartes.

BOSINSKI G. 1970 - Bemerkungen zu der Grabung D. Peyronys in La Micoque. Fundamenta, A2, p. 52-56, pl. 16-31.

BOURGUIGNON L. 1997 - Le Moustérien de type Quina: nouvelle définition d'une entité technique. Nanterre: Université de Paris X, 1997. 2t. 671p., Thèse N.D.: Ethn.

CAPITAN 1896 - Station acheuléenne de La Micoque. Revue de l'Ecole d'anthropologie, p. 406-416.

CAPITAN 1907 - Nouvelles fouilles à La Micoque. La Revue préhistorique Annales de Palethnologie, 2 , p. 1-9.

CHAUVET G. 1896 - Station quatemaire de La Micoque. Bulletin de la Société Archéologique et Historique de la Charente.

CHAUVET G., RIVIERE E. 1896 - Gisement quatemaire de La Micoque. C. R. de l'Académie des Sciences, 24 Août 1896 p. 284.

COUTIL L. 1905 - Similitude de certaines stations paléolithiques de la Dordogne, de la Charente, du Mâconnais et de l'Eure ; Compte-Rendu du Congrès Préhistorique de Périgueux.

DEBÉNATH A., DELPECH F., GENESTE J.M., RIGAUD J.Ph., TEXIER J.P. 1991 - Nouvelles recherches à La Micoque, Dordogne (France). In : Résumés du colloque commémoratif international de Miskolc (Hongrie). $5 \mathrm{p}$.

DEBÉNATH A., RIGAUD J.Ph. 1986 - Le gisement de La Micoque. In : Rigaud J.Ph. (Dir.), Informations archéologiques: circonscription d'Aquitaine ; Gallia Préhistoire, 29, p. 236-237.

FALGUÈRES C., BAHAIN J.J., SALEKI H. 1997 - U-Series and ESR Dating of Teeth from Acheulian and Mousterian Levels at La Micoque (Dordogne, France). Journal of Archaeological Science, 24, p. 537-545. 
FIEDLER L. 1997 - Älteres Paläolithikum aus dem Gebiet zwischen Mittelrhein, Main und Werra. In : Fiedler L. (Ed.), Archäologie der ältesten Kultur in Deutschland. Wiesbaden, p. 49-79 (Materialien zu Vor- und Frühgeschichte von Hessen 18).

FILZER P. 1969 - Pollenanalytische Untersuchungen der Höhlen- und Hangschichten. In : Wetzel R. ; Bosinski G. (Ed.): Die Bocksteinschmiede im Lonetal (Markung Rammingen, Kreis Ulm). Stuttgart, p. 169-205 (Veröffentlichungen des staatlichen Amtes für Denkmalpflege Stuttgart Reihe A Heft 15).

GENESTE J.M., BOËDA E., MEIGNEN L. 1990 - Identification de chaînes opératoires lithiques du Paléolithique ancien et moyen. Paléo, 2, p. 43-80.

GOUEDO J.M. 1999 - Le technocomplexe micoquien en Europe de l'Ouest et Centrale; exemples de trois gisements du sud-ouest du Bassin parisien, Vinneuf et Champlost (Yonne), Verrières-le-Buisson (Essonne). Lille : Université des Sciences et Technologies de Lille, 1999. 2t., 488 p. Thèse N.D.

HAUSER O. 1906-1907 - La Micoque (Dordogne), und ihre Resultate für die Kenntnis der paläolithischen Kultur. 1. Teil. Basel: Selbstverlag, 10p.

HAUSER O. 1908a - Fouilles scientifiques à La Micoque, à Laugerie Basse et au Moustier. L'Homme préhistorique, 6, p. $40-48$.

HAUSER O. 1908b - Fouilles scientifiques dans la Vallée de la Vézère, revu et complété. L Homme préhistorique, 6 , p. $40-48$

HAUSER O. 1916 - La Micoque. Die Kultur einer neuen Diluvialrasse.Leipzig : Verlag von Veit \& Comp., 58p., 10pl.

JÖRIS O. 2001 - Der spätmittelpaläolithische Fundplatz Buhlen (Grabungen 1966-69). Stratigraphie, Steinartefakte und Fauna des Oberen Fundplatzes. Universitätsforschungen zur prähistorischen Archäologie 73, Bonn : Verlag Dr. Rudolf Habelt GmbH, 178 p.

JÖRIS O. 2002 - Die aus der Kälte kamen... von der Kultur später Neandertaler in Mitteleuropa. Mitteilungen der Gesellschaft für Urgeschichte, 11, p. 3-32.

LAVILLE H. 1975 - Climatologie et chronologie du paléolithique en Périgord. Etudes Quatemaires 4, Editions du laboratoire de paléontologie humaine et préhistorique, Université de Provence, 422p.

LAVILLE H., RIGAUD J.Ph. 1976 - Le gisement de La Micoque. In : Laville H. ; Rigaud J.Ph. (Ed), Livret-Guide de l'excursion A4 Sud-Ouest (Aquitaine et Charente), IXe Congrès de l'UISPP, Paris, p. 49-57.

LUTTROPP A., BOSINSKI G. 1971 - Der altsteinzeitliche Fundplatz Reutersruh bei Ziegenhain in Hessen. Institut für Ur- und Frühgeschichte der Universität zu Köln, Köln-Wien : Böhlau Verlag, 94p, 215 pl.
MONCEL M.H. 1999 - Les assemblages lithiques du site Pléistocène moyen d'Orgnac 3 (Ardèche, Moyenne Vallée du Rhône, France). ERAUL 89, Liège, 446 p.

OBERMAIER H. 1908 - Die Steingeräte des französischen Altpaläolithikums. Mitteilungen der Prähistorischen Kommission der Kaiserlichen Akademie der Wissenschaft 2 Nr.1, Berlin, 125 p.

PATTE E. 1971 - L'industrie de La Micoque. L'Anthropologie, 75, p. 369-396.

PEYRONY D. 1908a - Etude comparée des deux niveaux quatemaires de La Micoque (Dordogne). Bulletin de la Société de Géographie de Bordeaux, 1908, p. 3-12.

PEYRONY D. 1908b - A propos des fouilles de La Micoque et des travaux récents parus sur ce gisement. Revue de l'Ecole d'anthropologie, 11, p. 380-382.

PEYRONY D. 1933 - La Micoque et ses diverses industries. In : XVe Congrès International d'Anthropologie et d'Archéologie Préhistorique (suite), Ve Session de l'Institut Intemational d'Anthropologie ; Paris 20-27 Septembre 1931, Paris, Librairie E. Nourry, Extrait, p. 1-6.

PEYRONY D. 1938 - La Micoque. Les fouilles récentes. Leur signification. Bulletin de la Société Préhistorique Française, 35, p.121 et 257-288.

RICHTER J. 1997 - Der G-Schichten-Komplex der Sesselfelsgrotte. Zum Verständnis des Micoquien. Quartärbibliothek Band 7, Sesselfelsgrotte III, Saarbrücken, $473 \mathrm{p}$.

RICHTER J. 2002 - Die 14C-Daten aus der Sesselfelsgrotte und die Zeitstellung des Micoquien/M.M.O. Germania, 80, p.1-22.

SCHWARCZ H.P. ; GRÜN R. 1988 - ESR Dating of Level L2/3 at La Micoque, Dordogne (France), Excavation of Debénath and Rigaud. Geoarchaeology, 3, p. 293-296.

SCHMID E. 1969 - Die sedimentanalytischen Untersuchungen der Ablagerungen. In : Wetzel R. ; Bosinski G. (Ed.) : Die Bocksteinschmiede im Lonetal (Markung Rammingen, Kreis UIm). Stuttgart, p. 207-223 (Veröffentlichungen des staatlichen Amtes für Denkmalpflege Stuttgart Reihe A Heft 15).

TEXIER J.P. ; BERTRAN P. 1993 - Nouvelle interprétation paléoenvironnementale et chronostratigraphique du site paléolithique de La Micoque (Dordogne). Implications archéologiques. Comptes Rendus de l'Académie des Sciences de Paris, 316, Série 2, p. 1611-1617.

TURQ A. 1989 - Approche technologique et économique du faciès Moustérien de type Quina: étude préliminaire. Bulletin de la Société Préhistorique Française, 86 , p. 244-256. 
TURQ A. 2000 - Paléolithique inférieur et moyen entre Dordogne et Lot. Paléo supplément 2, Les Eyzies-deTayac, $456 \mathrm{p}$.

VEIL St., BREEST K., HÖFLE H.C., MEYER H.H., PLISSON H., URBAN-KÜTTEL B., WAGNER G.A., ZÖLLER L. 1994 Ein mittelpaläolithischer Fundplatz aus der Weichsel-Kaltzeit bei Lichtenberg, Lkr. Lüchow-Danneberg. Zwischenbericht über die archäologischen und geowissenschaftlichen Untersuchungen 1987-1992. Germania, 72, p. 1-65, 3 pl.
WETZEL R., BOSINSKI G. 1969 - Die Bocksteinschmiede im Lonetal (Markung Rammingen, Kreis UIm). Veröffentlichungen des staatlichen Amtes für Denkmalpflege Stuttgart Reihe A Heft 1, Stuttgart, 2 Bände, 230 p., 166 pl.

WIEGERS F., SCHUCHHARDT C, HILZHEIMER M. 1913 Eine Studienreise zu den paläolithischen Fundstellen der Dordogne. Zeitschrift für Ethnologie, 45, p. 126-160. 